\title{
Onset of shear band localization by a local generalized eigenvalue analysis
}

\author{
Miguel Arriaga, Colin McAuliffe, Haim Waisman* \\ Department of Civil Engineering and Engineering Mechanics, Columbia University; 610 Seeley \\ W. Mudd Building; 500 West 120th Street, Mail Code 4709; New York, NY 10027
}

\begin{abstract}
Shear bands are material instabilities associated with highly localized intense plastic deformation zones which can form in materials undergoing high strain rates. Determining the onset of shear band localization is a difficult task and past work reported in the literature attempt to detect this instability by computing the eigenvalues of the acoustic tensor or by studying the linear stability of the perturbed governing equations. However, both methods have their limitations and are not suited for general rate dependent materials in multidimensions.

In this work we propose a novel approach to determine the onset of shear band localization and alleviate the limitations of the above mentioned methods.

Owing to the implicit mixed finite elements discretization employed in this work, we propose to cast the instability analysis as a generalized eigenvalue problem by employing a particular decomposition of the element Jacobian matrix. We show that this approach is attractive, as it is applicable to general rate dependent multidimensional cases where no special simplifying assumptions ought to be made.

To verify the accuracy of the proposed eigenvalue analysis, we first extend an analytical criterion by applying linear perturbation techniques to the continuous
\end{abstract}

*Corresponding author: waisman@ civil.columbia.edu (Haim Waisman) 
PDE model, considering an elastoplastic material with thermal diffusion and a non-linear Taylor-Quinney coefficient. While this extension is novel on its own, it requires strenuous derivations and is not easily extended to general multidimension applications. Hence, herein it is only used for verification purposes in 1D.

Numerical results on one-dimensional problems show that the eigenvalue analysis exactly recovers the instability point predicted by the analytical criterion with nonlinear Taylor Quinney coefficient. In addition, the proposed generalized eigenvalue analysis is applied on two-dimensional problems where propagation of the instability can be easily determined.

\section{Introduction}

\subsection{Background on Shear bands}

Shear bands are material instabilities associated with highly localized intense plastic deformation zones, in materials such as metals, which typically form under high strain rate loadings. These localized bands have also been observed in materials with non-associative flow laws like porous or granular materials, polymers, rocks and soils [1, 2].

High strain rate loading amplifies strain rate hardening effects observed in many structural materials, which has a stabilizing effect. In contrast, thermal softening destabilizes the material as heat produced by inelastic deformation lowers the material's flow stress. The reduced flow stress then leads to more intense plastic deformation where an instability may occur. The influence of strain hardening, strain rate hardening and thermal softening produces a complex equilibrium that depends strongly on the loading rate and the material properties.

If shear banding is modeled as an adiabatic process then the width of the shear band will tend towards zero and the numerical solution will be mesh-dependent [3-6]. However, if thermal diffusion is considered, an intrinsic length scale forms 
and acts as a localization limiter, which has been shown to lead to mesh insensitive results [7-9]. Other regularization techniques reported in the literature include strain gradient theories [10-12], which have been used in the context of shear bands in [13-15] and nonlocal methods[16-18]. Mesh alignment is another form of sensitivity that can be improved using mesh-free formulations [19, 20] or Isogeometric analysis [21].

This work proposes a method to determine the onset of shear band instability by a specially formulated local generalized eigenvalue analysis. To this end, the semi-discrete Jacobian is decomposed from its mixed finite element formulation following the derivation in [22].

It is shown that the method is applicable to general rate-dependent materials with complex material laws, can be easily applied to multidimensions and its implementation is straight forward.

Moreover, we show that this method will provide more reliable results in $2 \mathrm{D}$ when compared to an analytical criterion derived in the literature. Although similar techniques that compute the eigenvalues of the Jacobian matrix originated from the finite element discretization have been employed in other fields (e.g. stability of fluid dynamics [23] or thermal capillarity instability [24]), to the authors' knowledge, this methodology has never been employed to study local instabilities of shear bands.

Stability analysis of shear bands has been an active area of research. Among others, two main approaches on the study of stability involving shear bands are the linear perturbation method [8, 25,-34] and the eigenvalue analysis of the acoustic tensor [35-38]. This paper focuses on the linear perturbation method and not on the loss of ellipticity detected by the eigenvalues of the acoustic tensor since for a rate-dependent material the equations remain elliptical was shown by Lemonds and Needleman [39], "[...] although localization is a prominent feature of our results, the mathematical difficulties associated with loss of ellipticity do not arise. For the rate dependent constitutive relation used in this investigation, the gov- 
erning equations remain elliptic [...]". In other words, a rate-dependent material could make the equations remain elliptic, making the eigenvalue approach presented in this work more appropriate than the approach based on the eigenvalues of the acoustic tensor.

The Taylor-Quinney(TQ) coefficient determines the fraction of inelastic work that is converted to heat and in most studies in the literature has been assumed to be constant with a value of 0.9 . Nonetheless, recent studies indicate that the TQ coefficient is not constant and may depend on internal variables such as strain rate, plastic strain and temperature, among others. These studies assume that some portion of the plastic work goes into cold work, which is energy stored in defects within the metal's lattice [40, 41]. Following these studies we extend the derivation of Bai [26] to include a generic non-constant strain-rate dependent Taylor Quinney coefficient.

The functional non-linearity of the TQ coefficient is a very active field of research and proper satisfactory consensus on its formulation is yet to be found [40-43]. This paper does not attempt to provide a contribution to the field that studies the thermodynamic non-linearity of the Taylor-Quinney coefficient and its effect on the response of steel, but to illustrate the power of the proposed technique in analyzing an example functional form of TQ.

\subsection{Problem Statement}

The equations describing conservation of momentum and energy, as well as the elastic and inelastic constitutive relations and boundary conditions can be written as a set of coupled PDE's, and include the following. The Momentum Equation, which includes inertial effects but ignores body forces, is given by

$$
\rho \ddot{\mathbf{u}}=\nabla \cdot \sigma
$$

Here the displacement field is $\mathbf{u}$, the time is $t$, the stress tensor $\sigma$ and the material density $\rho$. A super imposed dot corresponds to a time derivative. The Elastic 
Constitutive Relation, in total form, is

$$
\sigma=\mathbf{C}^{\text {elas }}:\left(\varepsilon-\varepsilon^{p}\right)
$$

Where $\mathbf{C}^{\text {elas }}$ is the fourth-order elastic constitutive tensor, and the strain tensor has been additively decomposed into elastic and inelastic parts [44] as

$$
\varepsilon=\frac{1}{2}\left[\nabla \mathbf{u}+(\nabla \mathbf{u})^{T}\right]=\varepsilon^{e}+\varepsilon^{p}
$$

with $\varepsilon^{e}$ and $\varepsilon^{p}$ as the elastic and inelastic parts of the strain, respectively. The energy equation, which accounts for diffusion as well as heat production in proportion to the plastic work, $\sigma: \mathbf{D}^{p}[45]$, where $\mathbf{D}^{p}=\dot{\varepsilon}^{p}$ is the rate of deformation, is written as

$$
\rho c_{p} \dot{T}=\lambda \nabla^{2} T+\chi\left(\bar{\gamma}_{p}, \dot{\bar{\gamma}}_{p}, T, \ldots\right) \sigma: \mathbf{D}^{p}
$$

where $T$ is the temperature, and $\lambda, c_{p}$, and $\chi$ are the conductivity, specific heat and Taylor-Quinney coefficient, respectively. In this paper we assume a non constant form for $\chi$ which depends only on the plastic strain rate, such that

$$
\chi\left(\dot{\bar{\gamma}}_{p}\right)=\chi^{*} \eta\left(\dot{\bar{\gamma}}_{p}\right)
$$

where $\chi^{*}=0.9$ is the reference constant Taylor-Quinney coefficient and $\eta\left(\dot{\bar{\gamma}}_{p}\right)$ represents a general non-linear function that scales the reference coefficient.

This particular choice of the Taylor-Quinney function allows for a generalization of the stability analysis in the following section to a non-constant Taylor-Quinney coefficient. Note, however, that this formulation is still a significant simplification of the complex physics in such processes.

The rate of change of the equivalent plastic strain is defined as

$$
\dot{\bar{\gamma}}_{p}=\sqrt{\frac{2}{3} \mathbf{D}^{p}: \mathbf{D}^{p}}
$$


J2 plasticity is employed so that the inelastic part of the rate of deformation tensor is defined as

$$
\mathbf{D}^{p}=\frac{3}{2} \frac{\dot{\bar{\gamma}}_{p}}{\bar{\sigma}} \mathbf{S}
$$

where the deviatoric stress tensor $\mathbf{S}$ is

$$
\mathbf{S}=\sigma-\frac{1}{3} \operatorname{tr}(\sigma) \mathbf{I}
$$

and the effective stress is given by

$$
\bar{\sigma}=\sqrt{\frac{3}{2} \mathbf{S}: \mathbf{S}}
$$

The inelastic constitutive relation, given in terms of the effective stress, may be written as

$$
\bar{\sigma}=P(T) Q\left(\bar{\gamma}_{p}\right) R\left(\dot{\bar{\gamma}}_{p}\right)
$$

where $\bar{\gamma}_{p}$ is the equivalent plastic strain, which serves as a strain hardening parameter and the functions $P(T), Q\left(\bar{\gamma}_{p}\right)$ and $R\left(\dot{\bar{\gamma}}_{p}\right)$ depend on specific material models. The analysis presented henceforth will be general for any flow law that can be decomposed as in (10) [46]. An example of a flow law that respects these conditions is the Johnson-Cook material model [47], hereafter used for the numerical results of this paper.

The four equations (1), (2), (4), (10) along with the definition of the flow law describe the evolution of the four unknown fields of displacement, stress, temperature and plastic strain.

Lastly, the boundary conditions are 


$$
\begin{aligned}
\mathbf{u} & =\overline{\mathbf{u}} & & \text { on } & & \Gamma^{u} \\
T & =\bar{T} & & \text { on } & & \Gamma^{T} \\
\mathbf{n} \cdot \sigma & =\overline{\mathbf{t}} & & \text { on } & & \Gamma^{t} \\
\mathbf{n} \cdot \mathbf{q} & =\bar{q} & & \text { on } & & \Gamma^{q}
\end{aligned}
$$

Where $\overline{\mathbf{t}}$ and $\bar{q}$ are the prescribed traction and flux, respectively. This model considers small strains, and additionally neglects thermal strains and the thermoelastic contribution to the system energy. The effects of voids and micro-cracking that exist in shear bands [48] are also not modeled in this study.

\section{Linear perturbation analysis of the $1 \mathrm{D}$ shear band problem}

In this section, a linear perturbation analysis is conducted to predict the onset of shear band instability. This type of analysis is common in the literature and has,

for example, been carried out by Bai [26] under rigid plasticity assumptions, and by Ling and Belytschko [30] under adiabatic conditions. Herein a generalization and extension of the linear perturbation analysis is done to include elasto-plastic materials with thermal diffusion and a non-linear Taylor-Quinney coefficient.

Consider a 1D shear band model assuming a narrow strip of material in simple shear. In this case only shear components are modeled and hence all derivatives with respect to $x$ and $z$ are equal to zero. By restricting equations (1), (2), (4) and (10) to $1 \mathrm{D}$, a new set of governing equations is obtained. The momentum equation is reduced to

$$
\frac{\partial \tau}{\partial y}=\rho \frac{\partial^{2} u}{\partial t^{2}}
$$

where $\tau$ is the shear stress and $y$ is the spacial dimension considered. The elastic 
constitutive relation is given by

$$
\tau=G\left(\gamma-\gamma^{p}\right)
$$

with $G$ representing the shear modulus, $\gamma=\frac{\partial u}{\partial y}$ the shear strain and $\gamma^{p}$ the plastic shear strain. The energy equation is now expressed as

$$
\rho c_{p} \dot{T}=\lambda \frac{\partial^{2} T}{\partial y^{2}}+\chi\left(\dot{\gamma}^{p}\right) \tau \dot{\gamma}^{p}
$$

where $\dot{\gamma}^{p}$ is the rate of change of the plastic shear strain and the non-linear constitutive relation is given by

$$
\tau=P(T) Q\left(\gamma^{p}\right) R\left(\dot{\gamma}^{p}\right)
$$

here expressed as a function of the simple shear quantities $\tau, \gamma^{p}$ and $\dot{\gamma}^{p}$. Note that the model presented here is a simplified restriction of the full set of governing equations (section 1.2) to a $1 \mathrm{D}$ simple shear case. If the non-linear constitutive relation is directly derived from Eq. (10), then additional constants will appear in Eq. (18) due to the J2 plasticity assumptions. Without loss of generality, we discard these constants in the stability analysis by considering a 1D pure shear case. Nonetheless, the reader may refer to Appendix A for more details on the relation between the two models.

Following the linear perturbation method, let $\mathbf{s}_{0}(t)=\left[u_{0}(t), \sigma_{0}(t), T_{0}(t), \gamma_{0}^{p}(t)\right]$ be a solution of equations (15), (16), (17) and (18) at time $t$. The solution is perturbed by a small perturbation $\delta \mathbf{s}$ at $t=t_{0}$ such that

$$
\mathbf{s}(y, t)=\mathbf{s}_{0}\left(t_{0}\right)+\delta \mathbf{s}\left(t_{0}, y, t\right)
$$

where $\delta \mathbf{s} \ll \mathbf{s}_{0}$. 
The perturbation $\delta \mathbf{s}\left(t_{0}, y, t\right)$ is given by:

$$
\delta \mathbf{s}\left(t_{0}, y, t\right)=\hat{\mathbf{s}}_{0} e^{\omega\left(t-t_{0}\right)+i k y}
$$

where $\hat{\mathbf{s}}_{0}, \omega$ and $k$ are the amplitude, the rate of change and wave-number of the perturbation, respectively and $i=\sqrt{-1}$.

Strictly imaginary values of $\omega$ correspond to a pure oscillatory solution without growth or decay, whereas strictly real values of $\omega$ correspond to a monotonic solution, which can be asymptotically stable $(\operatorname{Re}[\omega]<0)$ or unstable $(\operatorname{Re}[\omega]>0)$. A combination of both is also possible, corresponding to an oscillatory mode, which can be stable or unstable depending on whether the eigenvalue's real part is negative or positive, respectively [49, 50]. If a solution $\mathbf{s}_{0}\left(t_{0}\right)$ has a positive value of $\operatorname{Re}[\omega]$, then it will be considered an instability point, since it corresponds to an exponential growth of the unstable mode given by the amplitude of the perturbation.

It is worth nothing that the relative inhomogeneity $\delta \mathbf{s} / \mathbf{s}_{0}$ might decrease if the rate of change of the homogeneous flow $\mathbf{s}_{0}$ is large enough, which according to Fressengeas and Molinari [29] justifies why instability and localization do not coincide.

The perturbation method is applied to the governing equations resulting in the fourth-order characteristic equation $R(\omega)$ presented in 21 . The reader may refer to Appendix B for the full derivation of the characteristic equation.

$$
R(\omega)=a_{4} \omega^{4}+a_{3} \omega^{3}+a_{2} \omega^{2}+a_{1} \omega+a_{0}=0
$$


where

$$
\begin{aligned}
& a_{0}=k^{4} \lambda Q_{0} \\
& a_{1}=k^{2}\left(k^{2} \lambda R_{0}+c_{p} Q_{0} \rho-\chi^{*} P_{0} \tau_{0}^{*}\right) \\
& a_{2}=\rho\left[\chi^{*} \dot{\gamma}_{0}^{p *} P_{0}+k^{2}\left(\lambda+c_{p} R_{0}\right)\right] \\
& a_{3}=\rho\left(c_{p} \rho+\frac{k^{2} \lambda R_{0}-\chi^{*} P_{0} \tau_{0}^{*}}{G}\right) \\
& a_{4}=\frac{c_{p} R_{0} \rho^{2}}{G}
\end{aligned}
$$

\section{with the non-linear TQ coefficient terms are given by}

$$
\chi_{0}=\chi^{*} \eta\left(\dot{\gamma}_{0}^{p}\right)=\chi^{*} \eta_{0},\left.\quad \frac{\partial \chi}{\partial \dot{\gamma}^{p}}\right|_{0}=\chi^{*} \eta^{\prime}\left(\dot{\gamma}_{0}^{p}\right)=\chi^{*} \eta_{0}^{\prime}
$$

the corrected stress and plastic strain-rate terms to account for contribution of the non-linear TQ coefficient as

$$
\tau_{0}^{*}=\left(\eta_{0}^{\prime} \dot{\gamma}_{0}^{p}+\eta_{0}\right) \tau_{0}, \quad \dot{\gamma}_{0}^{p *}=\eta_{0} \dot{\gamma}_{0}^{p}
$$

\section{and $P_{0}, Q_{0}$ and $R_{0}$ defined by}

$$
P_{0}=-\left.\frac{\partial \tau}{\partial T}\right|_{\mathbf{s}=\mathbf{s}_{0}} \quad ; \quad Q_{0}=\left.\frac{\partial \tau}{\partial \gamma^{p}}\right|_{\mathbf{s}=\mathbf{s}_{0}} \quad ; \quad R_{0}=\left.\frac{\partial \tau}{\partial \dot{\gamma}^{p}}\right|_{\mathbf{s}=\mathbf{s}_{0}}
$$

We note that $P_{0} \geq 0$ corresponds to thermal softening, $Q_{0} \geq 0$ corresponds to strain hardening and $R_{0} \geq 0$ corresponds to strain-rate hardening.

If the Taylor-Quinney coefficient is constant, then $\dot{\gamma}_{0}^{p *}=\dot{\gamma}_{0}^{p}, \tau_{0}^{*}=\tau_{0}$ and $\chi^{*}=\chi$. In this case it is interesting to note that, if rigid-plasticity is assumed by taking $G \rightarrow \infty$, then (21) is reduced to the same characteristic equation as in Bai [26]. On the other hand, if an adiabatic condition is assumed by taking $\lambda=0$, then (21) is reduced to the same characteristic equation as in Ling and Belytschko [30] (with the appropriate scaling to account for the restriction of $\mathrm{J} 2$ plasticity to $1 \mathrm{D})$, with the exception of the term that depends on $\dot{\gamma}_{0}^{p *}$ in $a_{2}$ which stems 
from the perturbation of the stress field in the energy equation and was included in our formulation (and also in Bai [26]). Hence, the formulation in this section generalizes previous work and expands the scope of stability analysis to include a non-linear Taylor-Quinney coefficient.

Following a similar non-dimensionalization as Bai [26], we define

$$
\tilde{\omega}=\frac{\lambda \omega}{c_{p} Q_{0}}
$$

Thus, the characteristic equation (21) can be rewritten with non-dimensional terms as follows

$$
\begin{aligned}
& \tilde{R}(\tilde{\omega})=\tilde{k}^{4}+\left(1-B+A \tilde{k}^{2}\right) \tilde{k}^{2} \tilde{\omega}+\left[C+(1+A) \tilde{k}^{2}\right] \tilde{\omega}^{2} \\
& \quad+\left[1+D\left(A \tilde{k}^{2}-B\right)\right] \tilde{\omega}^{3}+A D \tilde{\omega}^{4}=0
\end{aligned}
$$

with the non-dimensional parameters given by

$$
\begin{aligned}
\tilde{k}^{2} & =\frac{\lambda^{2} k^{2}}{\rho c_{p}^{2} Q_{0}} \\
A & =\frac{c_{p} R_{0}}{\lambda} \\
B & =\frac{\chi^{*} \tau_{0}^{*} P_{0}}{\rho c_{p} Q_{0}} \\
C & =\frac{\chi^{*} \lambda P_{0} \dot{\gamma}_{0} *}{\rho c_{p}^{2} Q_{0}} \\
D & =\frac{Q_{0}}{G}
\end{aligned}
$$

To proceed with the stability analysis, one has to determine the wave number $\tilde{k}$. To this end, two extreme cases of $\tilde{k} \rightarrow 0$ and $\tilde{k} \rightarrow \infty$ are considered first, which represent very long and very short wave-lengths, respectively. We also consider the general case for which $0<\tilde{k}<\infty$. Here, a value of $\tilde{k}$ which maximizes the roots of the characteristic equation is considered, since the maximum root will be the first to become positive. 
Case $\tilde{k} \rightarrow 0$. for very long wave-lengths the characteristic equation reduces to:

$$
\tilde{R}(\tilde{\omega})=C+(1-B D) \tilde{\omega}+A D \tilde{\omega}^{2}=0
$$

By applying Routh-Hurwitz methods [51, 52], the roots of the characteristic equation will have a positive real part only if

$$
B>\frac{1}{D} \quad \text { or } \quad \frac{\chi^{*} \tau_{0}^{*} P_{0}}{\rho c_{p}}>G
$$

Considering that $\chi^{*} \sim 1$ and for typical metals $\rho c \sim 10^{6} \mathrm{PaK}^{-1}$ and $P_{0} \sim 10^{6} \mathrm{PaK}^{-1}$, a value of shear stress larger than the shear modulus would be necessary for instability to occur with very long wave-lengths. However, this is not likely to happen in practice, since metals typically have a shear modulus $G$ a few orders of magnitude higher than the yield stress. Therefore, the current stress $\tau_{0}^{*}$ will also be smaller than $G$ preventing the instability of this mode.

Case $\tilde{k} \rightarrow \infty$. for very short wave-lengths, the characteristic equation will only have one finite root given by $\tilde{\omega}=-\frac{1}{A}$, and since this root is always negative, this case is always stable. This instability mode is controlled by thermal diffusion, since it generates the fourth-order terms on $\tilde{k}$, giving the finite root presented before. Moreover, in a short wave-lengths scenario, thermal diffusion will strongly dissipate heat, limiting any softening that originates from temperature rise.

If, however, thermal diffusion is set to zero, then the order of the $\tilde{k}^{2}$ terms in the characteristic equation is reduced and a new condition for stability, $B>1$, is obtained. This result is consistent with results presented for adiabatic conditions [30] where short wave-length perturbations are no longer stabilized by thermal diffusion and instability is feasible.

Case $0<\tilde{k}<\infty$. under general conditions, one can observe that the characteristic equation in 27) may have a positive real root since, following to Routh-Hurwitz 
methods, the coefficient of the $\tilde{\omega}$ term might become negative. Since the solution is stable for extreme values of $\tilde{k}$, which implies negative values for the roots, it is expected that there will be an intermediate value of $\tilde{k}$ that maximizes the root with maximum real part which will produce the first instability point.

The value of $\tilde{k}$ will be obtained using the same methodology as in the literature 8 , 26. 27], which will give a wave-number that maximizes the value of the root as

$$
\tilde{k}_{m}^{2}=\tilde{\omega}_{m} \frac{(B-1)-(1+A) \tilde{\omega}_{m}-A D \tilde{\omega}_{m}^{2}}{2\left(A \tilde{\omega}_{m}+1\right)}
$$

which by plugging back into the characteristic equation (27) gives

$$
4(1+A \tilde{\omega})\{C+\tilde{\omega}[1+D(A \tilde{\omega}-B)]\}=[1-B+\tilde{\omega}(1+A+A D \tilde{\omega})]^{2}
$$

Considering that $D \sim 10^{-2} \ll 1$ then (32) is reduced to

$$
4(1+A \tilde{\omega})(C+\tilde{\omega})=[1-B+\tilde{\omega}(1+A)]^{2}
$$

which is the same equation as the one obtained by Bai [26]. There it was shown that for a solution $\tilde{\omega}>0$ to exist, and assuming a value of $C \sim 10^{-10} \dot{\gamma}_{0}^{p} \ll 1$ (valid in practical applications to metals), the following criterion for instability is obtained

$$
B>1
$$

The physical meaning of this criterion is beyond the scope of this paper and is amply discussed in the literature. We refer the reader to Bai [26] for further information. Notice that in the present paper $B$ is different than in Bai's work, although it reduces to the same parameter for a constant Taylor Quinney coefficient. This condition was also obtained by Ling and Belytschko [30] for an adiabatic elastoplastic case, with constant Taylor-Quinney and considering the scaling for 1D restriction of $\mathrm{J} 2$ plasticity. 
To summarize, regardless of whether rigid-plasticity or adiabatic simplifications are used, the condition in (34) provides a good approximation of the instability point. Also, the parameter $C$ provided the characteristic equation with its rate dependency, which means that in regular metals the rate-sensitivity will not strongly influence the instability point.

\section{Proposed approach: Lyapunov stability of the discretized problem}

In this section, the stability of the system is analyzed by the indirect method of Lyapunov[50]. The aim is to determine the onset of shear band localization directly by a generalized eigenvalue analysis, applied to a decomposed variant of the element Jacobian matrix. The eigenvalue criterion is then compared with the analytical criterion obtained in section 2. It is shown that a direct eigenvalue approach circumvents the laborious process of deriving an analytical type criterion and no special restrictions/assumptions are required.

Firstly, consider the system of equations expressed in its autonomous form as

$$
\mathscr{L}(\dot{\mathbf{x}})=F(\mathbf{x})
$$

where $\mathbf{x}$ represents all non-constant variables of the problem, $\mathscr{L}$ represents a linear operator and $F(\mathbf{x})$ is a non-linear function of $\mathbf{x}$.

The variables $\mathbf{x}$ can be expressed as a sum of the equilibrium solution with a perturbation $\delta \mathbf{x}$ as:

$$
\mathbf{x}=\mathbf{x}_{0}+\delta \mathbf{x}
$$

Linearizing $F(\mathbf{x})$ by a Taylor Series Expansion around $\mathbf{x}_{0}$ and considering only the first order term gives

$$
F(\mathbf{x})=F\left(\mathbf{x}_{0}\right)+F^{\prime}\left(\mathbf{x}_{0}\right) \delta \mathbf{x}
$$


Thus, (35) can be written as

$$
\mathscr{L}\left(\dot{\mathbf{x}}_{0}+\delta \dot{\mathbf{x}}\right)=F\left(\mathbf{x}_{0}\right)+F^{\prime}\left(\mathbf{x}_{0}\right) \delta \mathbf{x}
$$

Since $\mathbf{x}_{0}$ is a solution point, $\mathscr{L}\left(\dot{\mathbf{x}}_{0}\right)=F\left(\mathbf{x}_{0}\right)$, which means that

$$
\mathscr{L}(\delta \dot{\mathbf{x}})=\mathbf{A} \cdot \delta \mathbf{x}
$$

where $\mathbf{A}=F^{\prime}\left(\mathbf{x}_{0}\right)$ is the linearized Jacobian matrix of $F(\mathbf{x})$ at $\mathbf{x}_{0}$. In our model, this matrix will be obtained from mixed finite element formulations, described later in this section. This process centers the stability analysis problem on the equilibrium point such that it becomes the origin, permitting the use of a Lyapunov indirect stability analysis[50]. Representing the operator $\mathscr{L}(\cdot)$ by a matrix $\mathbf{M}$ and considering a linear perturbation of the form

$$
\delta \mathbf{x}=\hat{\mathbf{x}} e^{\omega t}
$$

where $\omega \in \mathbb{C}$ is an eigenvalue and $\hat{\mathbf{x}}$ its corresponding eigenvector representing the mode shape of the perturbation, gives the following generalized eigenvalue problem

$$
\mathbf{A} \delta \mathbf{x}=\omega \mathbf{M} \delta \mathbf{x}
$$

Here $\mathbf{M}$ is the mass matrix and $\mathbf{A}=\mathbf{K}+\mathbf{G}$ represents the sum of the stiffness matrices associated with linear material behavior such as elasticity and thermal diffusion $(\mathbf{K})$ and the tangent stiffness matrices associated with material non-linear behavior $(\mathbf{G})$.

This method of stability analysis was proposed by Lyapunov[53] and has been referred to as the first method of Lyapunov or the indirect method of Lyapunov [50, 54].

Note that the indirect method of Lyapunov is equivalent to the linear perturbation method if the system of equations were merely ODEs. However, since the shear 
band is described by a system of PDEs, they also involve spacial derivatives in addition to time derivatives. If a wave-number were to be used to approximate these spacial derivatives, then both methodologies would generate the same characteristic equation. However, the focus of this work is to use the finite element method for the stability analysis. This means that the resulting wavelengths thus modeled will directly depend on the discretization and the shape functions chosen.

Similar to the linear perturbation method presented in section 2, the discrete Lyapunov stability analysis will produce a characteristic equation on the rate of growth of the perturbation, for which the roots will provide the stability of the system. However, unlike the linear perturbation method, the wave length is not solved for but is incorporated in the eigenvectors of the problem.

The following subsection describes the mixed finite element formulation for implementing the shear band problem and the setup of the generalized eigenvalue analysis. A stability condition is then derived directly from the eigenvalue problem and compared with the criterion obtained with the linear perturbation method.

\subsection{Finite Element formulation}

In this section, the governing equations for the shear band problem will be discretized using a mixed finite element formulation. This discretization will be used to set up the generalized eigenvalue problem for the stability analysis. Following Lyapunov stability analysis described earlier, the system of equations (15)-(18) are first reformulated in the following autonomous form

$$
\begin{aligned}
\rho \dot{\mathbf{v}} & =\nabla \cdot \sigma \\
\dot{\sigma} & =\mathbf{C}^{\text {elas }}:\left(\nabla^{s} \mathbf{v}-\frac{3}{2} \frac{g\left(\bar{\sigma}, T, \bar{\gamma}_{p}\right)}{\bar{\sigma}} \mathbf{S}\right) \\
\rho c_{p} \dot{T} & =\lambda \nabla^{2} T+\chi^{*} \eta(g) \bar{\sigma} g\left(\bar{\sigma}, T, \bar{\gamma}_{p}\right) \\
\dot{\bar{\gamma}}_{p} & =g\left(\bar{\sigma}, T, \bar{\gamma}_{p}\right)
\end{aligned}
$$


with the rate of deformation tensor given by the symmetric part of the velocity gradient or can directly be defined by taking the time derivative of Eq. (3), which reads

$$
\nabla^{s} \mathbf{v}=\frac{1}{2}\left[\nabla \mathbf{v}+(\nabla \mathbf{v})^{T}\right]
$$

To obtain the weak form in mixed formulation, each equation is pre-multiplied by an appropriate shape function $w_{v}, w_{\sigma}, w_{T}$ and $w_{\bar{\gamma}_{p}}$ for the momentum equation, the elastic constitutive equation, the energy equation and the inelastic constitutive equation, respectively. Then, integrating over the domain and applying the divergence theorem where appropriate, the weak form of the residual equations is obtained.

Specifically, the residual of the momentum equation (42) reads

$$
\mathbf{R}_{v}=\int_{\Omega} w_{\nu} \rho \dot{\mathbf{v}} \mathrm{d} \Omega+\int_{\Omega} \nabla w_{\nu} \sigma \mathrm{d} \Omega-\int_{\Gamma} w_{\nu} \sigma \cdot \mathbf{n} \mathrm{d} \Gamma
$$

The weak form of the residual of the elastic constitutive equation 443 is

$$
\mathbf{R}_{\sigma}=\int_{\Omega} w_{\sigma} \dot{\sigma} \mathrm{d} \Omega-\int_{\Omega} w_{\sigma} \mathbf{C}^{e l a s}:\left(\nabla^{s} \mathbf{v}-\frac{3}{2} \frac{g\left(\bar{\sigma}, T, \bar{\gamma}_{p}\right)}{\bar{\sigma}} \mathbf{S}\right) \mathrm{d} \Omega
$$

The residual of the energy equation (44) reads

$$
\mathbf{R}_{T}=\int_{\Omega} w_{T} \rho c_{p} \dot{T} \mathrm{~d} \Omega+\int_{\Omega} \nabla w_{T} \lambda \nabla T \mathrm{~d} \Omega-\int_{\Omega} w_{T} \chi^{*} \eta \bar{\tau} g \mathrm{~d} \Omega-\int_{\Gamma} w_{T} \lambda \nabla T \cdot \mathbf{n} \mathrm{d} \Gamma
$$

and finally, the weak form of the residual of the inelastic constitutive equation (45) is

$$
\mathbf{R}_{\bar{\gamma}_{p}}=\int_{\Omega} w_{\bar{\gamma}_{p}} \dot{\bar{\gamma}}_{p} \mathrm{~d} \Omega-\int_{\Omega} w_{\bar{\gamma}_{p}} g \mathrm{~d} \Omega
$$

These equations can be grouped into a residual vector $\mathbf{R}$ and a solution vector $\mathbf{x}$ 
with displacements, temperature, stress and plastic strain fields as

$$
\mathbf{x}=\left[\begin{array}{c}
\mathbf{v} \\
T \\
\sigma \\
\bar{\gamma}_{p}
\end{array}\right] \quad \mathbf{R}=\left[\begin{array}{c}
\mathbf{R}_{v} \\
\mathbf{R}_{T} \\
\mathbf{R}_{\sigma} \\
\mathbf{R}_{\bar{\gamma}_{p}}
\end{array}\right]
$$

The coupled nonlinear problem can then be stated

$$
\mathbf{R}\left(\mathbf{x}, \dot{\mathbf{x}}, \mathbf{u}_{i b v}\right)=0
$$

where $\mathbf{u}_{i b v}$ represents the initial and the boundary conditions, which for simplicity of notation will be dropped henceforth. To solve (52), the Newton method is applied, assuming a Taylor series expansion around $\mathbf{x}_{0}$ and $\dot{\mathbf{x}}_{0}$, such that

$$
\mathbf{R}(\mathbf{x}, \dot{\mathbf{x}})=\mathbf{R}\left(\mathbf{x}_{0}, \dot{\mathbf{x}}_{0}\right)+\left.\mathrm{d}_{\delta \mathbf{x}} \mathbf{R}(\mathbf{x}, \dot{\mathbf{x}})\right|_{\mathbf{x}_{0}, \dot{\mathbf{x}}_{0}}+\left.\mathrm{d}_{\delta \dot{\mathbf{x}}} \mathbf{R}(\mathbf{x}, \dot{\mathbf{x}})\right|_{\mathbf{x}_{0}, \dot{\mathbf{x}}_{0}}
$$

where $\mathrm{d}_{\delta \mathbf{x}}(\cdot)$ and $\mathrm{d}_{\delta \dot{\mathbf{x}}}(\cdot)$ represent the Gâteaux differentials in the $\delta \mathbf{x}$ and $\delta \dot{\mathbf{x}}$ directions, respectively, which are defined as

$$
\begin{aligned}
& \mathrm{d}_{\delta \mathbf{x}} \mathbf{R}(\mathbf{x}, \dot{\mathbf{x}})=\left.\frac{\mathrm{d}}{\mathrm{d} \varepsilon} \mathbf{R}(\mathbf{x}+\varepsilon \delta \mathbf{x}, \dot{\mathbf{x}})\right|_{\varepsilon=0}=-\mathbf{A} \cdot \delta \mathbf{x} \\
& \mathrm{d}_{\delta \dot{\mathbf{x}}} \mathbf{R}(\mathbf{x}, \dot{\mathbf{x}})=\left.\frac{\mathrm{d}}{\mathrm{d} \varepsilon} \mathbf{R}(\mathbf{x}, \dot{\mathbf{x}}+\varepsilon \delta \dot{\mathbf{x}})\right|_{\varepsilon=0}=\quad \mathbf{M} \cdot \delta \dot{\mathbf{x}}
\end{aligned}
$$

Considering that $\mathbf{R}(\mathbf{x}, \dot{\mathbf{x}})=0$, gives

$$
-\mathbf{R}\left(\mathbf{x}_{0}, \dot{\mathbf{x}}_{0}\right)=\mathbf{M} \cdot \delta \dot{\mathbf{x}}-\mathbf{A} \cdot \delta \mathbf{x}
$$

It is interesting to note that (55) represents both the equation for instability analysis by the Indirect method of Lyapunov and the equations for Newton's method for solving the nonlinear problem with an implicit scheme. To emphasize this point, 
note that if the initial point $\left(\mathbf{x}_{0}, \dot{\mathbf{x}}_{0}\right)$ is a solution, then its residual will vanish and (55) will translate into the Lyapunov stability equation in (41). On the other hand, if the residual doesn't vanish, then a typical formulation for Newton iterations is obtained.

$$
\mathbf{J} \delta \mathbf{x}=-\mathbf{R}
$$

where $\mathbf{J}$ corresponds to the Jacobian matrix (often called Tangent Stiffness matrix) and is computed by applying the Gâteaux derivative to the appropriate residual. Depending on the time discretization chosen, the Jacobian matrix can be expressed as a linear combination of the matrices $\mathbf{M}$ and $\mathbf{A}$. If the process is assumed to be quasi-static, the Mass matrix $\mathbf{M}$ will simply become zero. Furthermore, for a Backward Euler discretization in time, the Jacobian matrix is given by

$$
\mathbf{J}=\frac{1}{\Delta t} \mathbf{M}-\mathbf{A}
$$

where $\Delta t$ is the time step used to advance the solution. Note that the time integration scheme will affect the Jacobian matrix used to obtain a solution at a particular time step but not the matrices $\mathbf{M}$ and $\mathbf{A}$ used for the stability analysis. This happens because $\mathbf{M}$ and $\mathbf{A}$ are taken after a converged solution at a given time is reached and are therefore independent from the integration scheme.

The block structure of the matrix $\mathbf{M}$ is given by

$$
\mathbf{M}=\left[\begin{array}{cccc}
\mathbf{M}_{v} & 0 & 0 & 0 \\
0 & \mathbf{M}_{\sigma} & 0 & 0 \\
0 & 0 & \mathbf{M}_{T} & 0 \\
0 & 0 & 0 & \mathbf{M}_{\bar{\gamma}_{p}}
\end{array}\right]
$$


and the matrix $\mathbf{A}$ by

$$
\mathbf{A}=\left[\begin{array}{cccc}
0 & \mathbf{K}_{v \sigma} & 0 & 0 \\
\mathbf{K}_{\sigma v} & \mathbf{K}_{\sigma \sigma} & \mathbf{G}_{\sigma T} & \mathbf{G}_{\sigma \bar{\gamma}_{p}} \\
0 & \mathbf{G}_{T \sigma} & \mathbf{K}_{T T}+\mathbf{G}_{T T} & \mathbf{G}_{T \bar{\gamma}_{p}} \\
0 & \mathbf{G}_{\bar{\gamma}_{p} \sigma} & \mathbf{G}_{\bar{\gamma}_{p} T} & \mathbf{G}_{\bar{\gamma}_{p} \bar{\gamma}_{p}}
\end{array}\right]
$$

The inner blocks in $\mathbf{M}$ and $\mathbf{A}$ are presented in Appendix C. The subscripts on each block indicate the fields which are coupled, thus the pair $(i, j)$ represents the variation in the $j$ direction of the residual associated with field $i$.

Since the energy equation includes a non-linear Taylor-Quinney coefficient (not derived in McAuliffe and Waisman [22]), the non-linear terms $\mathbf{G}$ in the $\mathbf{A}$ matrix are given by

$$
\begin{aligned}
& \mathbf{G}_{T \sigma}=\chi^{*} \int_{\Omega} \mathbf{N}_{T}^{T}\left(\frac{\partial \eta}{\partial g} \frac{\partial g}{\partial \bar{\sigma}} g+\eta \frac{g}{\bar{\sigma}}+\eta \frac{\partial g}{\partial \bar{\sigma}}\right) \bar{\sigma} \frac{\partial \bar{\sigma}}{\partial \sigma} \mathbf{N}_{\sigma} \mathrm{d} \Omega \\
& \mathbf{G}_{T T}=\chi^{*} \int_{\Omega} \mathbf{N}_{T}^{T}\left(\frac{\partial \eta}{\partial g} g+\eta\right) \bar{\sigma} \frac{\partial g}{\partial T} \mathbf{N}_{T} \mathrm{~d} \Omega \\
& \mathbf{G}_{T \bar{\gamma}_{p}}=\chi^{*} \int_{\Omega} \mathbf{N}_{T}^{T}\left(\frac{\partial \eta}{\partial g} g+\eta\right) \bar{\sigma} \frac{\partial g}{\partial \bar{\gamma}_{p}} \mathbf{N}_{\bar{\gamma}_{p}} \mathrm{~d} \Omega
\end{aligned}
$$

\subsection{Application to a $1 D$ example}

To illustrate and compare the discrete Lyapunov stability analysis with the linear perturbation method, we show a stability analysis on a 1D discrete example. Similar to the analysis presented in section 2, the $1 \mathrm{D}$ problem is expressed in simple shear quantities $\tau$ and $\gamma$.

Accounting for the well known Babuska-Brezzi condition [55-57] in mixed finite element formulations, the shape functions for each field must be chosen with care. To this end, we choose $C^{0}$ shape functions for velocity and temperature fields and 
piecewise continuous functions for the stress and equivalent plastic strain fields. This results in an element with 6 degrees of freedom $\left(v_{1}, v_{2}, \tau, T_{1}, T_{2}\right.$ and $\left.\bar{\gamma}_{p}\right)$, as depicted in Figure 1 .

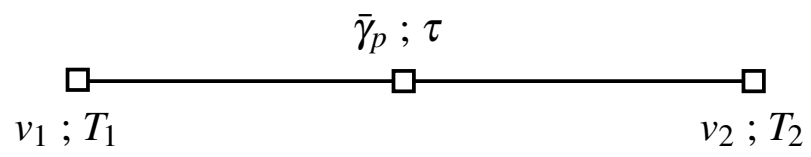

Figure 1: Representation of 1D element degrees of freedom. Squares represent nodes with the corresponding active fields.

Following the formulation in Section 3.1, the element matrices $\mathbf{M}^{e}$ and $\mathbf{A}^{e}$ are

$$
\mathbf{M}^{e}=h\left[\begin{array}{llllll}
\rho \frac{2}{6} & \rho \frac{1}{6} & & & \\
\rho \frac{1}{6} & \rho \frac{2}{6} & & & \\
& & 1 & & & \\
& & & \rho c_{p} \frac{2}{6} & \rho c_{p} \frac{1}{6} & \\
& & & \rho c_{p} \frac{1}{6} & \rho c_{p} \frac{2}{6} & \\
& & & & 1
\end{array}\right]
$$

and

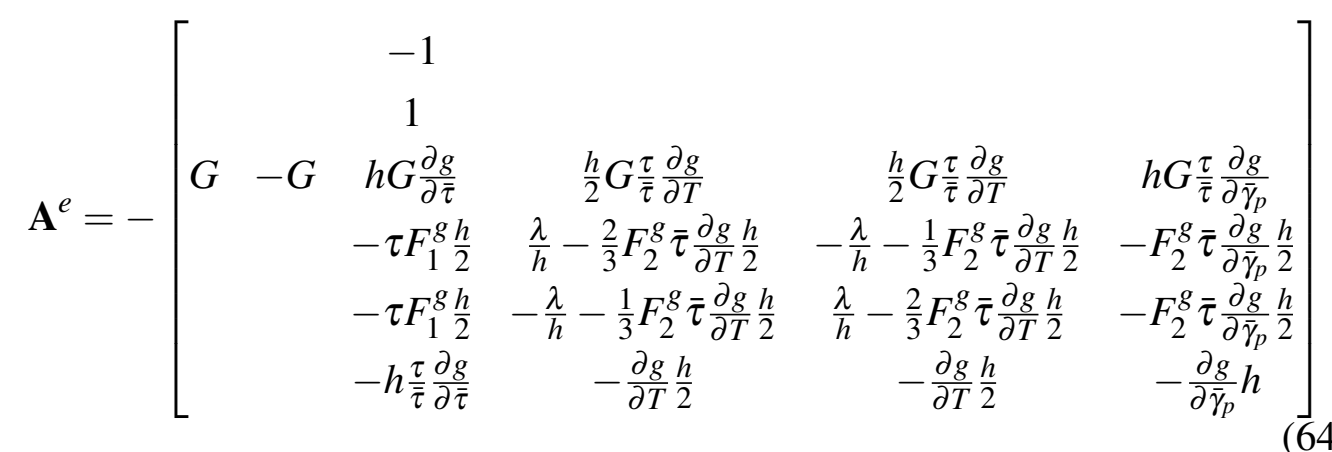

where $h$ is the element size and $\bar{\tau}$ is the effective shear stress. For simplicity, the non-linear fields are considered to be constant in each element and hence approx- 
imated by the following scalars

$$
\begin{aligned}
& F_{1}^{g}=\chi^{*}\left(\frac{\partial \eta}{\partial g} \frac{\partial g}{\partial \bar{\tau}} g+\eta \frac{g}{\bar{\tau}}+\eta \frac{\partial g}{\partial \bar{\tau}}\right) \\
& F_{2}^{g}=\chi^{*}\left(\frac{\partial \eta}{\partial g} g+\eta\right)
\end{aligned}
$$

For analysis of the characteristic equation it is assumed that $\frac{\tau}{\bar{\tau}}=1$, which corresponds to positive stresses. Considering

$$
\bar{\tau}=P(T) Q\left(\bar{\gamma}_{p}\right) R\left(\dot{\bar{\gamma}}_{p}\right) \quad \Rightarrow \quad \dot{\bar{\gamma}}_{p}=g=R^{-1}\left(\frac{\bar{\tau}}{P Q}\right)
$$

the derivatives of $g$ are given in terms of the derivatives of the flow law as

$$
\begin{gathered}
\frac{\partial g}{\partial \bar{\tau}}=\frac{1}{P Q R^{\prime}}=\frac{1}{R_{0}} \\
\frac{\partial g}{\partial \bar{\gamma}_{p}}=-\frac{Q^{\prime}}{Q} \frac{R}{R^{\prime}}=-\frac{Q_{0}}{R_{0}} \\
\frac{\partial g}{\partial T}=-\frac{P^{\prime}}{P} \frac{R}{R^{\prime}}=\frac{P_{0}}{R_{0}}
\end{gathered}
$$

Since the chosen element has six degrees of freedom, the final non-dimensional characteristic equation, $\tilde{R}^{h}(\tilde{\omega})$, obtained from (41) should also be of order six, that is

$$
\tilde{R}^{h}(\tilde{\omega})=a_{0}+a_{1} \tilde{\omega}+a_{2} \tilde{\omega}^{2}+a_{3} \tilde{\omega}^{3}+a_{4} \tilde{\omega}^{4}+a_{5} \tilde{\omega}^{5}+a_{6} \tilde{\omega}^{6}=0
$$

where

$$
\begin{aligned}
& a_{0}=a_{1}=0 \\
& a_{2}=\alpha \beta \gamma \\
& a_{3}=(\alpha+\beta) \gamma+\alpha C \\
& a_{4}=\gamma+\alpha(1+\beta \delta)+C \\
& a_{5}=1+(\alpha+\beta) \delta \\
& a_{6}=\delta
\end{aligned}
$$


The non-dimensional parameters are defined similarly to 27) and are given by

$$
\begin{aligned}
& \alpha=\left(A K^{2}-B\right) / A \\
& \beta=(1-B) / A \\
& \gamma=A K^{2} \\
& \delta=A D
\end{aligned}
$$

with

$$
\begin{aligned}
\tilde{\omega} & =\frac{\lambda \omega}{c_{p} Q_{0}} \\
K^{2} & =12 \frac{\lambda^{2}}{h^{2} \rho c_{p}^{2} Q_{0}} \\
A & =\frac{c_{p} R_{0}}{\lambda} \\
B & =\chi^{*}\left(\eta_{0}+\eta_{0}^{\prime} \dot{\gamma}_{0}^{p}\right) \frac{\tau_{0} P_{0}}{\rho c_{p} Q_{0}} \\
C & =\frac{\chi^{*} \eta_{0} \lambda P_{0} \dot{\gamma}_{0}^{p}}{\rho c_{p}^{2} Q_{0}} \\
D & =\frac{Q_{0}}{G}
\end{aligned}
$$

It is immediately noticeable that there are two null roots of the characteristic equation. As expected, the first zero eigenvalue is associated with a rigid body motion, which has an eigenvector given by $\phi_{1}=[1,1,0,0,0,0]^{T}$.

Also, the eigenvector $\phi_{2}=\left[0,0,0, \frac{Q_{0}}{P_{0}}, \frac{Q_{0}}{P_{0}}, 1\right]^{T}$ is associated with the second null eigenvalue. This eigenvector shows a change in temperature that is proportional to the change in plastic strain. The constant of proportionality is the relation between the softening of the stress due to a temperature change $\left(P_{0}\right)$ and the change in stress due to strain hardening $\left(Q_{0}\right)$. This eigenvalue corresponds to a situation where thermal softening and strain-hardening are perfectly balanced, producing a zero energy mode. Note, however, that this only happens due to the linearization considered, since the eigenvector will change with the solution.

It is worth mentioning that there is yet another eigenvalue that is simple to obtain, for which the eigenvector corresponds to a pure temperature gradient given by 
$\phi_{3}=[0,0,0,-1,1,0]^{T}$. The eigenvalue for this mode is given by

$$
\tilde{\omega}_{3}=-\alpha \frac{c_{p} Q_{0}}{\lambda}
$$

where $\frac{\lambda}{c_{p} Q_{0}}$ is the non-dimensional time. This eigenvalue will become positive (and therefore correspond to an instability mode) when the source parameter $B$ overcomes the thermal diffusion and rate hardening represented by $A K^{2}$, which is

$$
B>A K^{2} \quad \Rightarrow \quad \chi^{*}\left(\eta+\frac{\partial \eta}{\partial g} g\right) \tau_{0} P_{0}>\frac{12}{h^{2}} \lambda R_{0}
$$

This mode will generally not dominate the instability unless this element is used with adiabatic conditions (i.e. $\lambda=0$ ), in which case it will become unstable as soon as there is a non-zero source term. For this reason, in the adiabatic case, it is necessary to remove the pure-gradient mode from the solution space when performing the eigenvalue analysis numerically.

Applying the Routh-Hurwitz analysis on (70) we conclude that for instability to occur it is necessary that either $\alpha<0$ and/or $\beta<0$. The condition of $\alpha<0$ has already been discussed and is associated with a temperature gradient instability. The condition of $\beta<0$ corresponds to

$$
B>1
$$

and is exactly the same condition derived from the perturbation analysis on the strong-form equations in the previous section, Eq. (34). This result shows the equivalence between the two approaches and is verified numerically in section 4.

\section{Numerical Results}

The first set of results is used to validate the generalized eigenvalue analysis by comparison with the analytical criterion on a one dimensional problem, consid- 
ering different strain rates and a varying Taylor-Quinney coefficient. It is shown that the two methods exactly match in identifying the instability point.

The second set of results is used to investigate the performance of the numerical eigenvalue criterion on more complicated two dimensional problems.

Anand et al. [25] developed an analytical criterion for 2D considering adiabaticity and rigid plasticity assumptions. In their result, the wave-length and direction did not affect the stability condition, which led to the same instability criterion as the one presented here for 1D. Ling and Belytschko [30] also obtained this instability criterion for an adiabatic case but without the rigid-plasticity assumption. However, while the time-scale of the problem is very small, the thermal gradients are high enough for the diffusion term to have a magnitude of the same order as the source term in the vicinity of the shear band [22]. Therefore, it is of physical interest to conduct a stability analysis with the effects of diffusion included. This provides a motivation for attempting to compare the adiabatic analytical criterion with a criterion that includes thermal diffusion.

Note however that a generalization of the analytical criterion to include thermal diffusion in multi-dimensions will prove a significant challenge. This happens not only due to the existence of a new parameter (thermal conductivity) that influences stability, but especially because the introduction of the Laplacian on temperature will increase the order of the characteristic equation and make the stability depend on the wave-length. Conversely, applying the eigenvalue methodology is straight forward and avoids any simplifying assumptions like adiabaticity or rigidplasticity. Therefore, the adiabatic analytical criterion will be compared with the general eigenvalue criterion.

We therefore consider a couple of examples in 2D: (i) a benchmark example, where a square plate is stretched by uniaxial loading and a shear band develops in a 45 degree angle, and (ii) a notched plate problem (similar to the Kalthoff problem [58]), where an impact load is applied on a notched plate. 
The mixed finite element formulation is implemented in FEAP [59]. PETSc [60] is used to solve the nonlinear system of equations and LAPACK [61] is used for the eigenvalue problem.

The stability condition given by (77) will henceforth be referred to as the "Analytical" criterion and the condition in (78) will be referred to as the "Eigenvalue" criterion.

The equations were non-dimensionalized to safeguard against spurious numerical errors in the eigenvalue computation.

\subsection{Example in a $1 D$ rod}

\subsubsection{Problem description}

In this section a 1D steel rod is considered. The 1D formulation used is an idealized version of the Torsional Kolsky Bar experiment [62-65], which is commonly used to study a pure shear deformation without necking effects [66, 67]. It is shown numerically that the stability condition from the linear perturbation method agrees well with the eigenvalue analysis. The simulations are carried out for different strain rates with constant and varying Taylor-Quinney coefficients. The instability point defined in (34) and restated here as

$$
1-B<0
$$

coincides with the eigenvalue criterion when

$$
\max (\operatorname{Re}[\omega])>0
$$

where $\omega$ is the set of eigenvalues obtained from a generalized eigenvalue analysis and presented in 41 . 


\subsubsection{Mesh description and material model}

The rod used for computing the 1D results is discretized with varying element size to capture the shear band localization.

Considering symmetry, the relative position of the elements $(p)$ from the center to one edge (half-rod) can be defined by the following function

$$
p(\bar{x})= \begin{cases}S_{0} \bar{x} & \text { for } \bar{x} \leq \beta \\ S_{0} \bar{x}+\left(1-S_{0}\right)\left(\frac{\bar{x}-\beta}{1-\beta}\right)^{P} & \text { for } \bar{x}>\beta\end{cases}
$$

where $\bar{x}$ the relative node number, $P$ is the exponent used for varying the element size, $\beta$ is the percentage of nodes concentrated at the center of the rod with constant element size and $S_{0}$ is the slope for the central elements, defined as:

$$
S_{0}=\frac{d_{e}}{L} N
$$

where $L$ and $N$ are respectively the length and the number of nodes of the half-rod and $d_{e}$ defines the constant element size in the center of the rod.

In the following sections, the results are presented for $N=201, \beta=0.25, P=5$, $\frac{d_{e}}{L}=4 \times 10^{-4}$ and $L=0.5 \times 10^{-3} \mathrm{~m}$. All values refer to the half-rod, which means that the final length will be $10^{-3} \mathrm{~m}$. The total number of nodes will be 401 since the central node is repeated. Figure $2 \mathrm{a}$ shows the relative position of the nodes in the half-rod as a function of the relative node number and figure $2 \mathrm{~b}$ shows the relative element size also as a function of the relative node number.

The material considered for the 1D problem is a 4340 Steel with a Johnson-Cook constitutive law [47] given in (81). The parameters associated with this material are presented in Tables 1 and 2 .

$$
\tau=\left[A_{s}+B_{s}\left(\gamma^{p}\right)^{N}\right]\left[1-\left(\frac{T-T_{0}}{T_{m}-T_{0}}\right)^{m}\right]\left[1+c \ln \left(\frac{\dot{\gamma}^{p}}{\dot{\gamma}_{r}^{p}}\right)\right]
$$




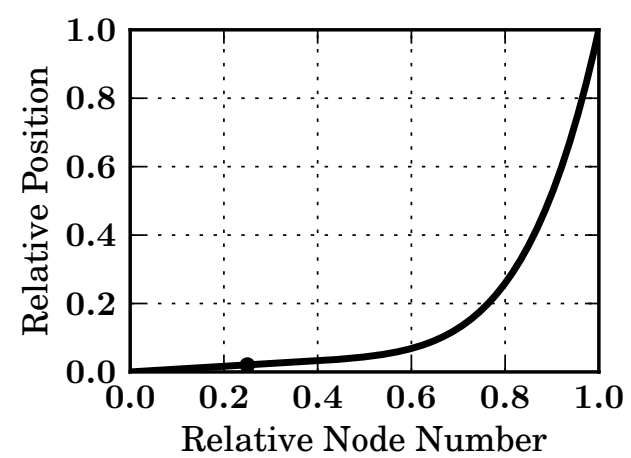

(a) Position of nodes

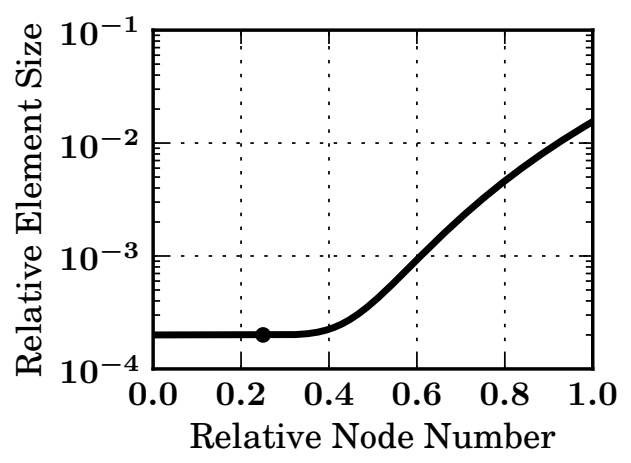

(b) Element Sizes

Figure 2: Representation of a 1D right half-rod. The black dot marks $\beta$

Table 1: Material properties for 4340 Steel

\begin{tabular}{lccc}
\hline Property Name & Symbol & Value & Unit \\
\hline Mass density & $\rho$ & 7830 & $\mathrm{~kg} / \mathrm{m}^{3}$ \\
Specific heat & $c_{p}$ & 477 & $\mathrm{~J} / \mathrm{kgK}$ \\
Thermal conductivity & $\lambda$ & 38 & $\mathrm{~W} / \mathrm{mK}$ \\
Young's modulus & $E$ & 200 & $\mathrm{GPa}$ \\
Poisson's ratio & $\mu$ & 0.29 & - \\
Shear modulus & $G$ & 77.5 & $\mathrm{GPa}$ \\
Taylor-Quinney coefficient & $\chi$ & 0.9 & - \\
\hline
\end{tabular}

Table 2: Johnson-Cook parameters for 4340 Steel

\begin{tabular}{lccc}
\hline Property Name & Symbol & Value & Unit \\
\hline Yield shear stress & $A_{s}$ & 457.3 & $\mathrm{MPa}$ \\
Shear stress hardening parameter & $B_{s}$ & 294.4 & $\mathrm{MPa}$ \\
Strain hardening parameter & $N$ & 0.26 & - \\
Reference Temperature & $T_{0}$ & 298 & $\mathrm{~K}$ \\
Melting Temperature & $T_{m}$ & 1793 & $\mathrm{~K}$ \\
Thermal softening exponent & $m$ & 1.03 & - \\
Strain-rate hardening parameter & $c$ & 0.014 & - \\
Reference strain-rate & $\dot{\gamma}_{r}^{p}$ & 1.0 & $1 / \mathrm{s}$ \\
\hline
\end{tabular}


The non-linearity of the Taylor-Quinney coefficient was set as proposed by Vural et al. [43] and described in (5] with the nonlinear scaling factor given by

$$
\eta\left(\dot{\gamma}^{p}\right)=\frac{1}{2}\left[1+\tanh \left(S_{A} \ln \frac{\dot{\gamma}^{p}}{\dot{\gamma}_{A}}\right)\right]
$$

Here $S_{A}$ is a scaling factor that describes the range of transition between the isothermal and the adiabatic cases and $\dot{\gamma}_{A}$ is the critical strain rate at which the contributions of heat generation and of heat loss are comparable (see Vural et al. [43] for more details). The following results are presented for $S_{A}=0.6$ and $\dot{\gamma}_{A}=27 \mathrm{~s}^{-1}$. Note that, given that the current FEM formulation already includes thermal diffusion, the use of this law will not produce physically meaningful results. However, the strong non-linearity of this scaling factor will allow for a good benchmark problem between the analytical and the eigenvalue criteria.

An hyperbolic secant type of imperfection was considered at the center of the rod which will scale the material parameter $A_{s}$ and $B_{s}$ by the factor

$$
\eta_{i m p}\left(r_{n}\right)=1-\alpha_{r e d}\left(\frac{2}{e^{r_{n}}+e^{-r_{n}}}\right)
$$

where $\alpha_{\text {red }}$ is the reduction of the material parameters in percentage at the center of the imperfection and $r_{n}$ is the normalized distance given by

$$
r_{n}(x, y)=\frac{\operatorname{abs}\left(x-x_{0}\right)}{r_{0}}
$$

with $x_{0}$ representing the center of the imperfection and $r_{0}$ its radius. For the problem studied $\alpha_{\text {red }}=0.01, r_{0}=10 \mu \mathrm{m}$ and $x_{0}=L / 2=500 \mu \mathrm{m}$.

The analysis is performed on a range of nominal strain rates between $10^{1} \mathrm{~s}^{-1}$ and $10^{5} \mathrm{~s}^{-1}$. Figure 3 shows the stress-strain behavior for different strain-rates with both constant and non-linear Taylor-Quinney coefficient. Considering the case of constant Taylor-Quinney coefficient represented by solid lines, it is shown that (for the range of strain-rates considered) a higher value of strain-rate implies a larger 
value of yield stress and an earlier collapse in terms of nominal strain. Note however, that the peak stress has little variation which will be shown to influence the instability point. By introducing Vural's [43] varying Taylor-Quinney coefficient model, the peak stress is delayed. On the other hand, as expected, large values of strain-rate show no difference between the cases of constant and non-linear Taylor-Quinney.

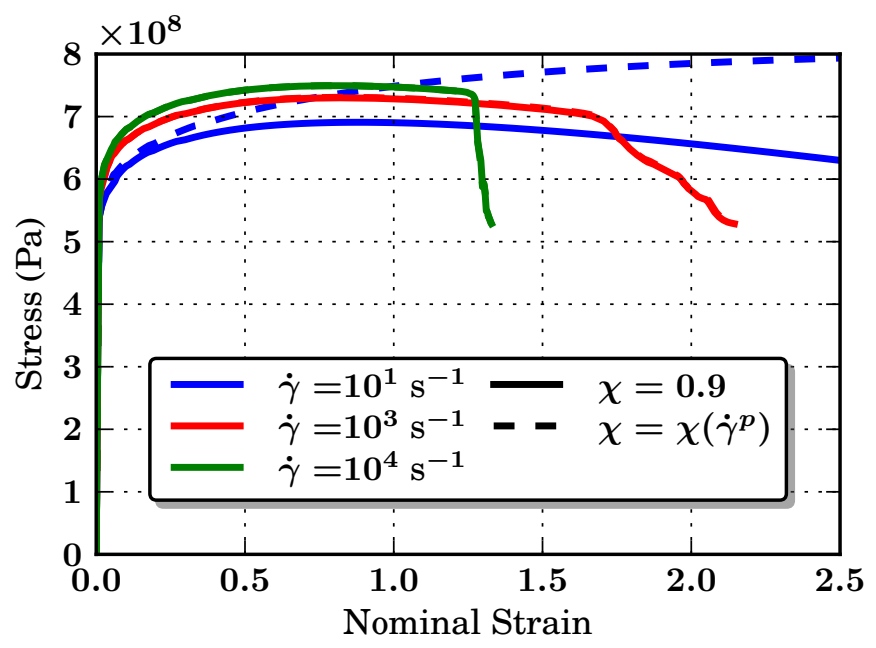

Figure 3: Stress-strain curves for several strain-rates. Solid and dashed lines represent constant and variable Taylor-Quinney coefficients, respectively. Note that no softening occurs, within the strain limits in the figure, for low strain rates with variable Taylor-Quinney coefficient.

\subsubsection{Discussion of results}

Figure 4 shows the computation of both the analytical (Eq. 77) and the eigenvalue (Eq. 78) stability criteria for the central point of the 1D rod. The study is carried out at four different nominal strain rates, considering a non-constant Taylor-Quinney coefficient. The gray line represents the stress at the center of the rod and the black and blue lines correspond to the analytical and eigenvalue criteria, respectively. It can be observed that the two stability criteria coincide and give identical results. 


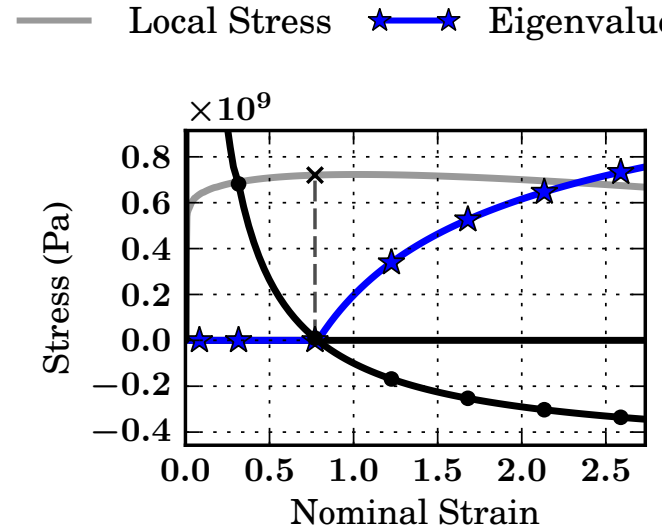

(a) Strain Rate: $10^{2} \mathrm{~s}^{-1}$

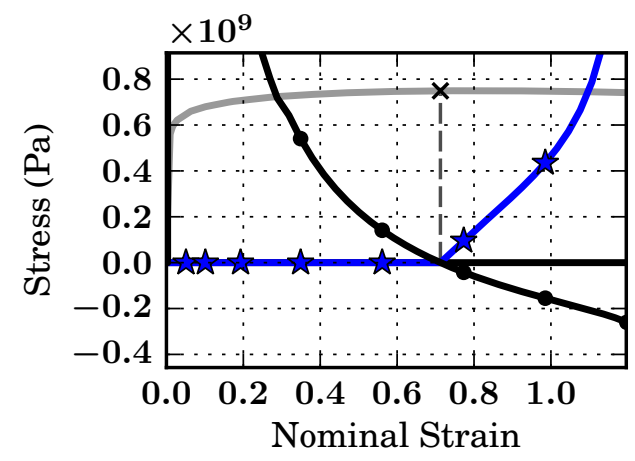

(c) Strain Rate: $10^{4} \mathrm{~s}^{-1}$ $\leftrightarrow$ Analytical $\quad \times \quad \operatorname{Re}[\omega] \geq 0$

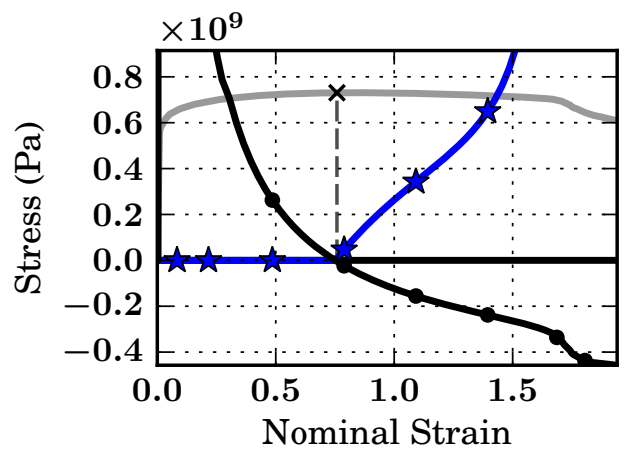

(b) Strain Rate: $10^{3} \mathrm{~s}^{-1}$

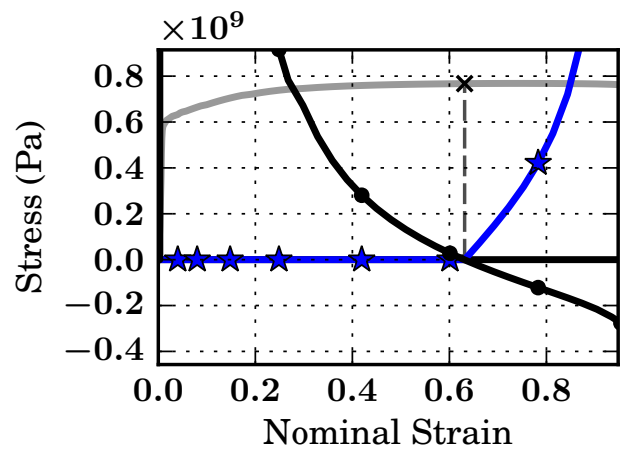

(d) Strain Rate: $10^{5} \mathrm{~s}^{-1}$

Figure 4: Stability analysis of 1D rod (center point) for non-constant Taylor-Quinney coefficient, at different strain rates. The analytical criterion is given by $1-B$ as given by Eq. 77 and the eigenvalue criterion is given by $\max (\operatorname{Re}[\omega])$ as shown in Eq. 78 . 
Figure 5 shows the nominal strain for the instability points and peak stress as a function of strain-rate. It is interesting to observe that the instability point obtained with a non-constant Taylor-Quinney model gives similar trend as the maximum stress criteria. As expected, the instability point and the peak stress are significantly delayed by the choice of Taylor-Quinney coefficient, at low strain-rates. This happens because lower values of $\eta\left(\dot{\gamma}^{p}\right)$, for smaller strain-rates, reduce the source term in the energy equation, which is the main driver of instability.

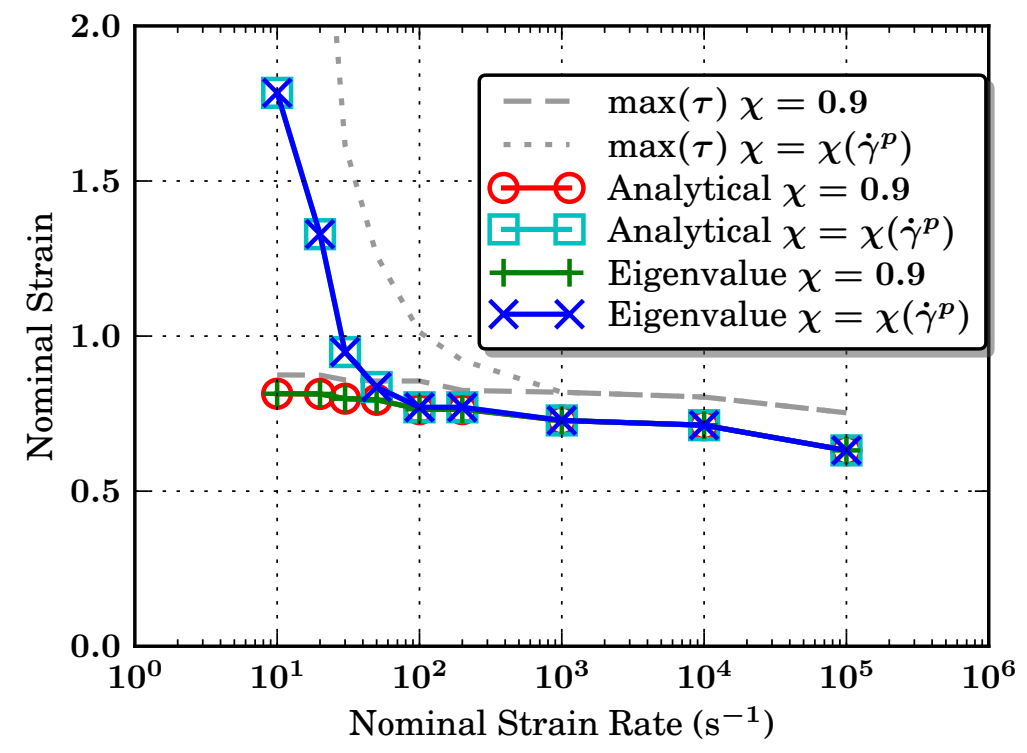

Figure 5: Instability points for both constant and non-constant Taylor-Quinney coefficients.

For the 1D models there are an average difference of 8 data points between the instability and the maximum stress, the minimum is 1 and the maximum is 45 . If we disregard the cases with non-constant TQc and very low strain-rates, the average is 3 data-points and the maximum is 8 . On the other hand, two types of analysis switch their stability at the same data-point.

Note that even though the nominal strain-rate is constant, locally the plastic strainrate is not. This means that the instability calculation has to take into account this variation by including it into the Residual and the Jacobian of each element as 
presented in section 3.1. In other words, the instability is influenced locally by the non-linear behavior of the Taylor-Quinney coefficient. This effect can be observed in figures 6 and 7 where the non linear factor $\eta\left(\dot{\gamma}^{p}\right)$ (given in Eq. 82) is plotted along the rod for different nominal strains.

Figures 6 and 7 show that, at the moment of stress collapse, the value of $\eta\left(\dot{\gamma}^{p}\right)$ goes to zero as the analysis evolves. This indicates that not only the plastic deformation is accumulating in the center of the rod, the localization process unloads the regions outside the shear band. By comparing the two figures it can be observed that the width of the shear band reduces as the nominal strain rate increases which is consistent with the results of Dodd and Bai [68].

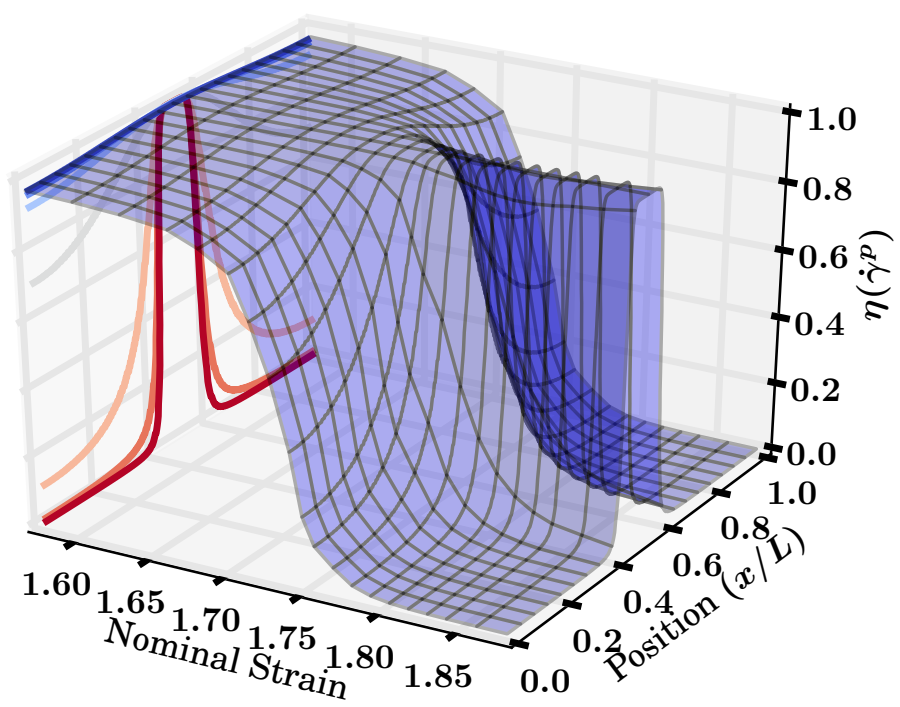

Figure 6: Local behavior along the rod of the non-linear Taylor-Quinney coefficient for a nominal strain-rate of $10^{3} \mathrm{~s}^{-1}$.

\subsection{Application to $2 D$}

We investigate the application of the eigenvalue criteria to two dimensional problems. Similar to 1D, 4340 steel is considered and a Johnson-Cook material law is 


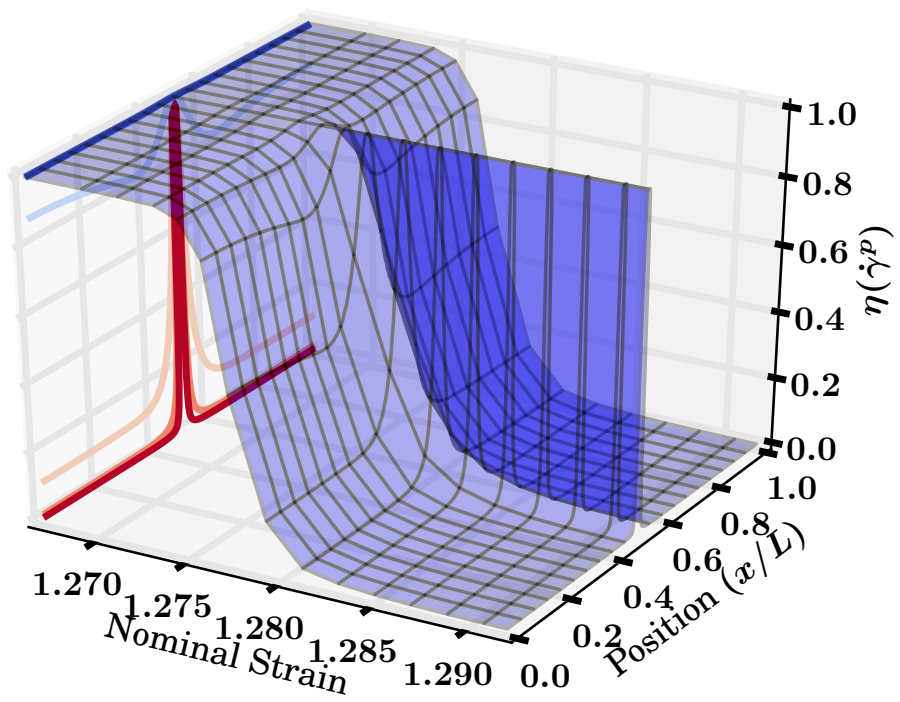

Figure 7: Local behavior along the rod of the non-linear Taylor-Quinney coefficient for a nominal strain-rate of $10^{4} \mathrm{~s}^{-1}$.

used with most of the parameters the same as those in 1D, presented in tables 1 and 2. Since the problem is $2 \mathrm{D}$, shear stress and plastic shear strain quantities are replaced with effective stress and equivalent plastic strain, respectively. These are standard terms defined in typical J2-Plasticity. The stress parameters $A$ and $B$ are given by:

$$
A=792 \mathrm{MPa} \quad B=510 \mathrm{MPa}
$$

Neumann boundary conditions for the Temperature field are applied on all edges and plane strain conditions are assumed. Since the main purpose of this study is to provide understanding on the behavior of the instability criteria, the small strain simplification is used without loss of generality.

\subsubsection{Benchmark example: $45^{\circ}$ shear band under uniform tension}

Consider a steel square plate as shown in figure 8 with $\mathrm{H}=10 \mathrm{~mm}$. 
An hyperbolic secant type of imperfection is used to scale by $\eta_{i m p}\left(r_{n}\right)$ the parameters $A$ and $B$, with $\eta_{\text {imp }}\left(r_{n}\right)$ given by equation 83 , and

$$
r_{n}(x, y)=\frac{\sqrt{\left(x-x_{0}\right)^{2}+\left(y-y_{0}\right)^{2}}}{r_{0}}
$$

with $x_{0}$ and $y_{0}$ representing the center of the imperfection and $r_{0}$ its radius. For the problem studied $\alpha_{\text {red }}=0.01, r_{0}=1 \mathrm{~mm}$ and $x_{0}=y_{0}=0 \mathrm{~mm}$.

The plate is stretched uniaxially with velocity control as shown in figure 9 with $v_{r}=20 \mathrm{~m} / \mathrm{s}$ and $t_{r}=50 \mu \mathrm{s}$.

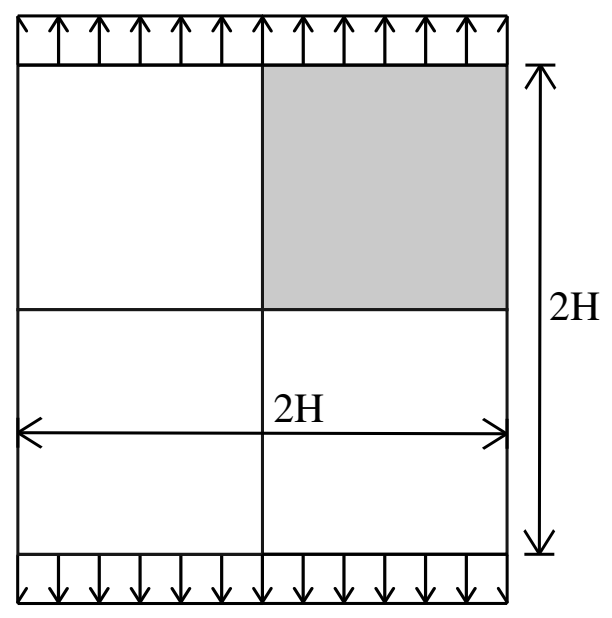

Figure 8: Full geometry of the Benchmark problem.

By symmetry considerations, only a quarter of the structure is modeled. The mesh consists of 40x40 square elements, modeled by an irreducible mixed-finite element formulation with $\overline{\mathrm{B}}$-bar to reduce shear-locking effects.

The velocity and temperature fields are discretized with bilinear $C^{0}$ continuous functions and the stress and equivalent plastic strain are bilinear $C^{-1}$ continuous. Details on the numerical implementation can be found in McAuliffe and Waisman [22, 69] and Berger-Vergiat et al. [21].

Figure 10 shows the behavior of the analytical and eigenvalue instability criteria 


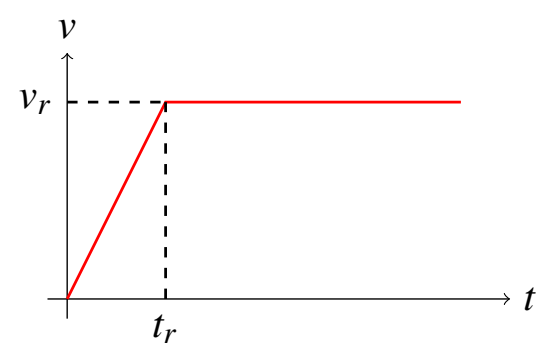

Figure 9: The velocity profile.

for the material point located at the center of plate (bottom-left corner). Even though both methods predict instability at a similar point in time, they are no longer coincident, which suggests that the trigger for instability differs between them.

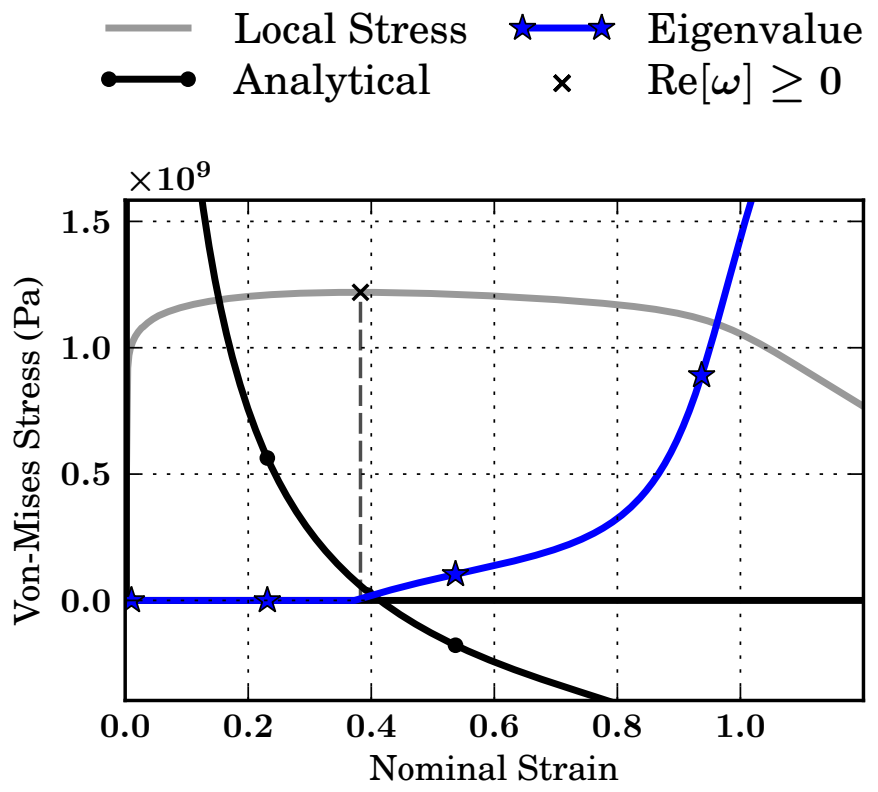

Figure 10: Evolution of instability for 2D model

Figure 11 shows the equivalent plastic strain $(11 \mathrm{~b}$ and $11 \mathrm{~d})$ and the eigenvalue instability condition for the 2D benchmark example $(11 \mathrm{a}$ and $11 \mathrm{c})$. Figures $11 \mathrm{a}$ and $11 \mathrm{~b}$ refer to the moment where the center of the plate (lower-left corner) 
becomes unstable which, as can be observed in figure 10, occurs at a nominal strain of $\varepsilon=0.37$. Figures $11 \mathrm{c}$ and $11 \mathrm{~d}$ show the same quantities at a later moment when $\varepsilon=1.1$. Here, the shear band has already developed along the diagonal and the instability region has spread through the shear band width.

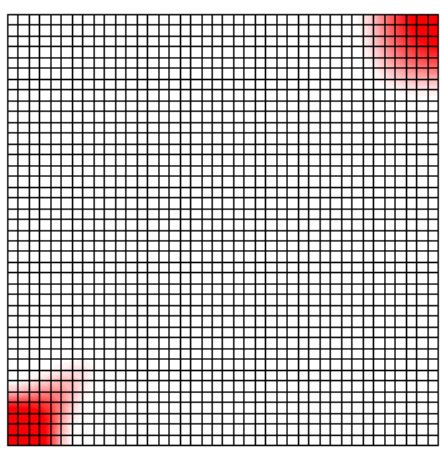

(a) Eigenvalue instability: $\varepsilon=0.37$

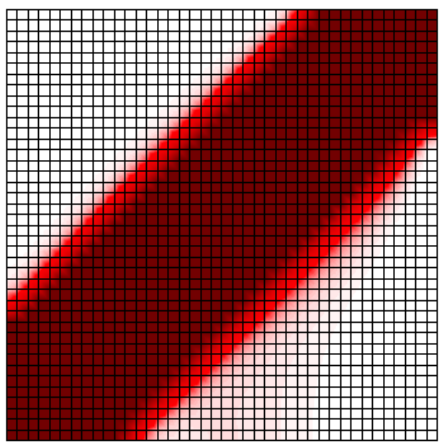

(c) Eigenvalue instability: $\varepsilon=1.1$

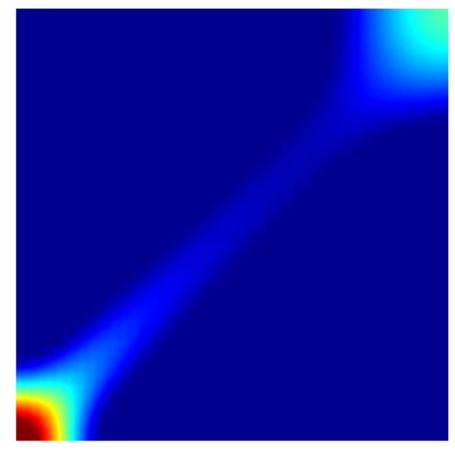

EQPS

0.44

0.43

(b) Equivalent plastic strain: $\varepsilon=0.37$

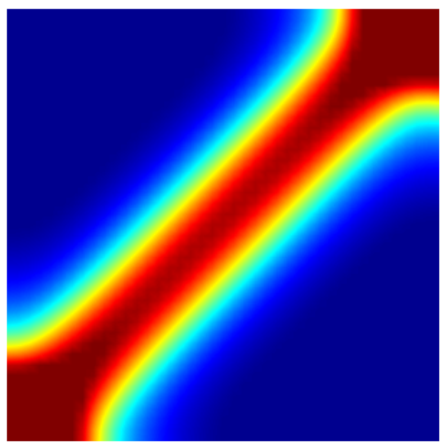

EQPS

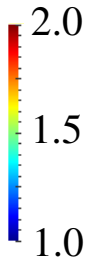

(d) Equivalent plastic strain: $\varepsilon=1.1$

Figure 11: Equivalent plastic strain (EQPS) and Eigenvalue instability condition at the first detected instability point $(\varepsilon=0.37)$ and at a later stage for which the the shear-band is fully developed $(\varepsilon=1.1)$. The Eigenvalue plots shown in red indicate the local instability.

Note that the equivalent plastic strain in figure $11 \mathrm{~b}$ is significantly more uniform when compared with figure $11 \mathrm{~d}$ In fact, shortly after the initial instability, shown in figure $11 \mathrm{~b}$, the entire plate becomes unstable, since the instability criteria are 
strongly dependent on the value of equivalent plastic strain. This is to be expected since, before localization, any point in the plate that is approaching a peak stress (i.e. softening becoming dominant) has the potential for localization and is therefore unstable. Similarly to buckling instability, where the path that is followed by the solution depends on material imperfections and/or asymmetry of the loading [70], shear band localization is also triggered by an inhomogeneity on the solution, which in the present case is due to an initial material imperfection.

Figure $11 \mathrm{c}$ shows that the eigenvalue stability condition is able to identify which part of the structure is undergoing unstable deformation since, as localization develops, the regions outside the shear band become stable again. Figure 12 shows that at a nominal strain of 1.1, the eigenvalue criterion indicates instability only in the region where the equivalent plastic strain has localized. In contrast, the analytical criterion fails to differentiate the localized region of the plate. Later, as the plastic deformation concentrates more intensely in the shear band, the analytical criterion tends toward the localized zone.

We refer the reader to the work of Ling and Belytschko [30], who proposed the multidimensional criterion for adiabatic shear bands that corresponds exactly to $B=1$ and also studied the post-localization stage.

The analytical criterion does not account for the stabilizing effect of diffusion, and therefore the unstable region predicted by it is typically larger than the unstable region predicted by the eigenvalue criterion. Additionally, note that when $\dot{\bar{\gamma}}_{p} \rightarrow 0$ (elastic behavior) the value of the parameter $A \rightarrow+\infty$ for a material law like Johnson-Cook, which has a stabilizing effect since the upper limit of the maximum root of the characteristic equation becomes zero [26]. However, considering that for the JC material law used $\dot{\bar{\gamma}}_{p}>0$ for $\bar{\sigma} \neq 0$ (although it can be very small), then a very large (but not infinite) value of A will allow for the criterion to still identify the existence of a true and valid positive real root (i.e. the criterion is still valid), but the magnitude of the root will be very close to zero, making its influence negligible. 
Hence, it is clear that the limitations of the analytical criterion can be overcome by the eigenvalue stability analysis.

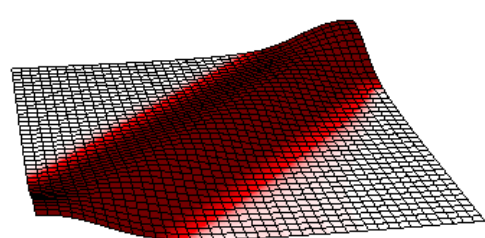

(a) Eigenvalue: $\varepsilon=1.1$

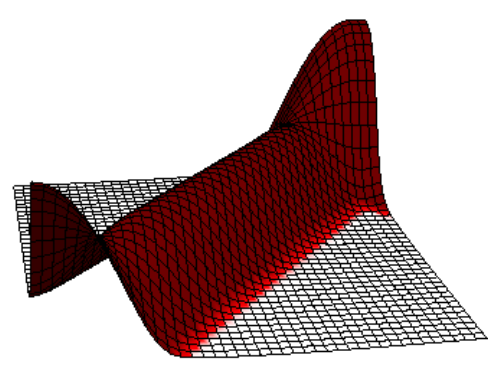

(c) Eigenvalue: $\varepsilon=2.1$

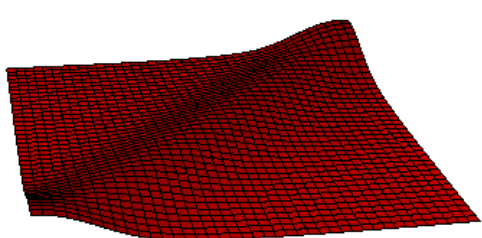

(b) Analytical: $\varepsilon=1.1$

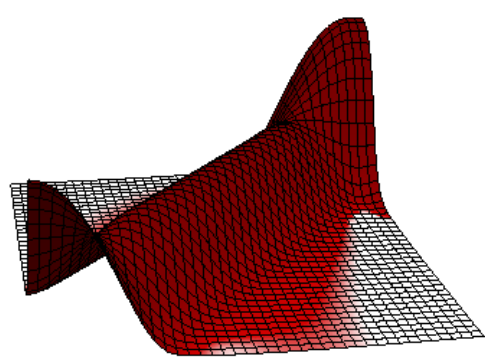

(d) Analytical: $\varepsilon=2.1$

Figure 12: Comparison between the Eigenvalue criterion and the Analytical criterion at certain points in time. The red color indicates where the local condition for instability is met and the displacement in the $z$ direction is proportional to the equivalent plastic strain.

\subsubsection{Notched plate problem: projectile impact onto a notched steel plate}

The following example models a notched steel plate impacted by a projectile. This problem is similar to the Kalthoff problem [58], that has been frequently studied with finite elements [71, 72] and mesh-free methods [3]. The geometry of the plate is shown in figure 13 with $\mathrm{H}=2 \times 10^{-5} \mathrm{~m}$, since the main goal is to model the notch tip. The impact load on the bottom half of the plate will be modeled by imposing a horizontal velocity boundary condition as shown in figure 9 with $v_{r}=200 \mathrm{~m} / \mathrm{s}$ and $t_{r}=10 \mathrm{~ns}$. 
By symmetry considerations, only the top half of the plate is analyzed as shown in figure 14a with symmetric boundary conditions applied at the bottom edge. The material parameters are the same as those used for the benchmark $45^{0}$ shear band problem, however, herein no material imperfection is introduced.

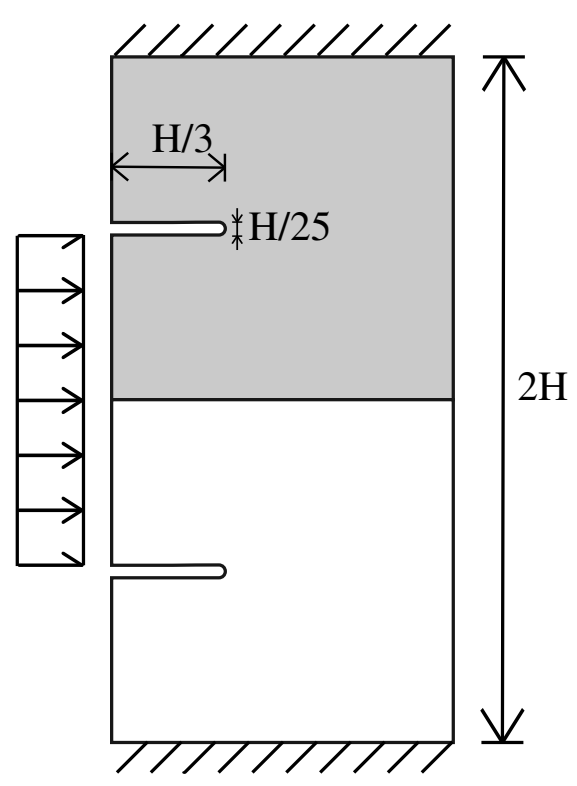

Figure 13: Full geometry of the notched problem.

Figure $14 \mathrm{~b}$ shows the Von-Mises stress at three points on the plate as a function of the nominal strain. It is clear from the figure that the peak stresses at points $P_{A}$ and $P_{B}$ occur in distinct points in time. The peak stress for $P_{A}$ is reached at an approximate time of $20 \mathrm{~ns}$, whereas for $P_{B}$ it is reached at approximately $65 \mathrm{~ns}$. Additionally, the stress drop at point $P_{0}$ will occur approximately $35 \mathrm{~ns}$ after the beginning of the analysis. However, note that this stress drop is not a collapse due to extreme softening but an elastic unloading that results from the propagation of the shear band along the center of the plate.

Figure 15 shows the real part of maximum eigenvalue and the analytical criterion for points $P_{A}, P_{B}$ and $P_{0}$. It is demonstrated that the stability criteria studied will consistently predict the peak stress after which localization might occur. How- 


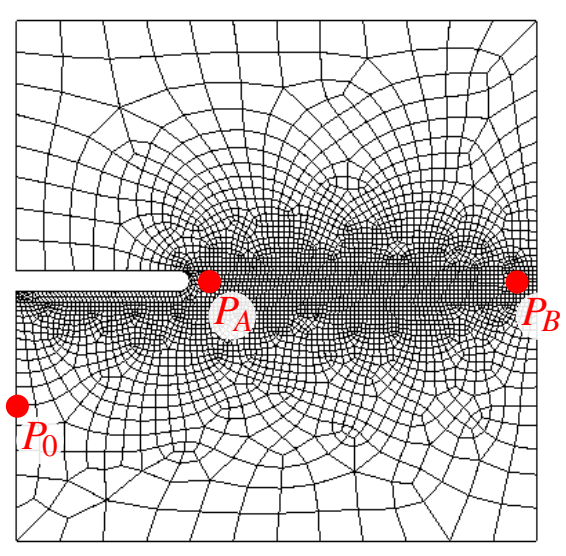

(a) 2D model for a notched plate

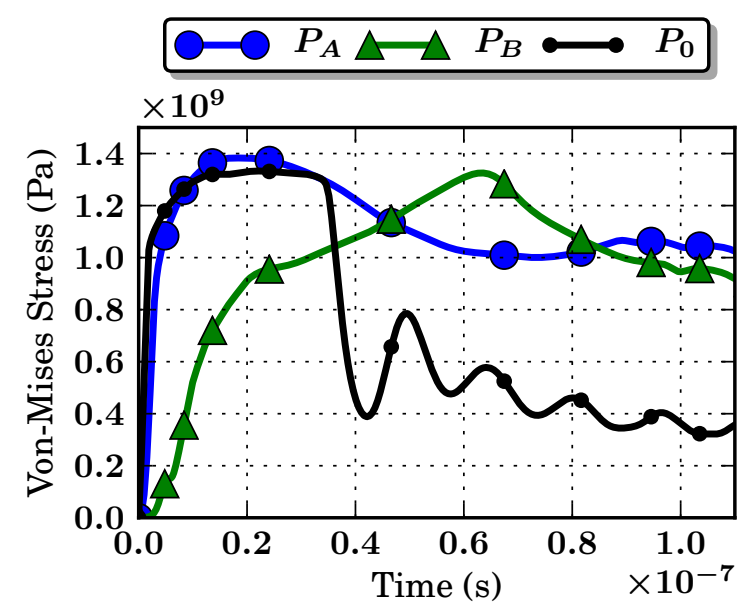

(b) Von-Mises stress evolution at points $P_{A}, P_{B}$ and $P_{0}$.

Figure 14: Impact onto a notched steel plate

ever, note that the existence of softening is not a necessary condition for instability since, as can be observed in figure 15c, the mild softening that occurs in $P_{0}$ after the peak stress and before the stress drop is not accompanied by an unstable eigenvalue.

As mentioned before, even though the analytical criterion and the eigenvalue criterion are in good agreement, they don't match exactly. Specifically, the first sign of instability is generally observed sightly earlier in the case of the eigenvalue analysis. Also, in the late stages of the analysis, the eigenvalue criterion predicts that the material points $P_{A}$ and $P_{B}$ regain stability while the analytical criterion does not. The latter difference between the two criteria (as discussed in section 4.2.1) is visible due to the higher accuracy of the eigenvalue analysis in delimiting the unstable region. In the present case the unstable region is narrow and since, as shown in section 4.2.1 elements may regain their stability as the analysis progresses, after approximately $90 \mathrm{~ns}$, the unstable region measured by the eigenvalue will no longer include points $P_{A}$ and $P_{B}$, which will not be the case for the analytical (adiabatic) criterion. 


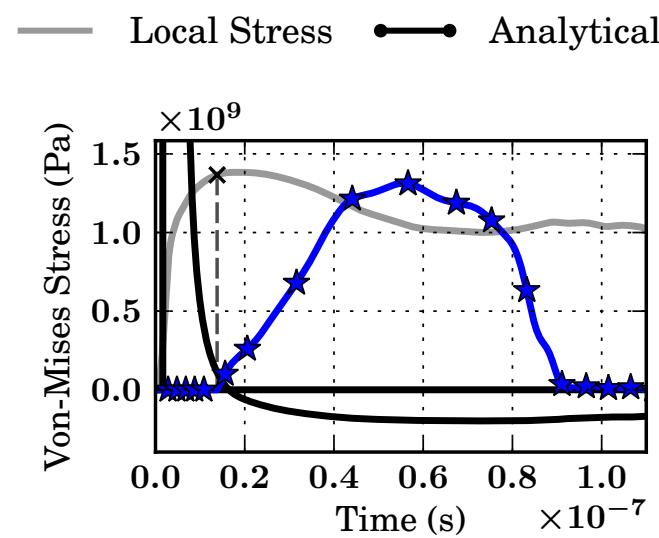

(a) Point $P_{A}$
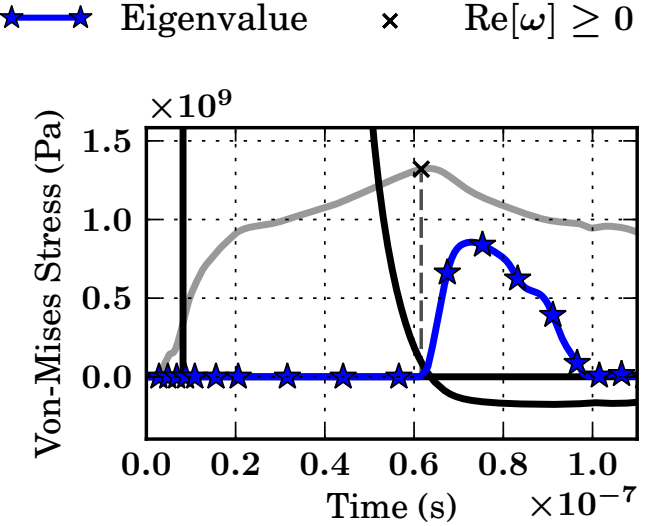

(b) Point $P_{B}$

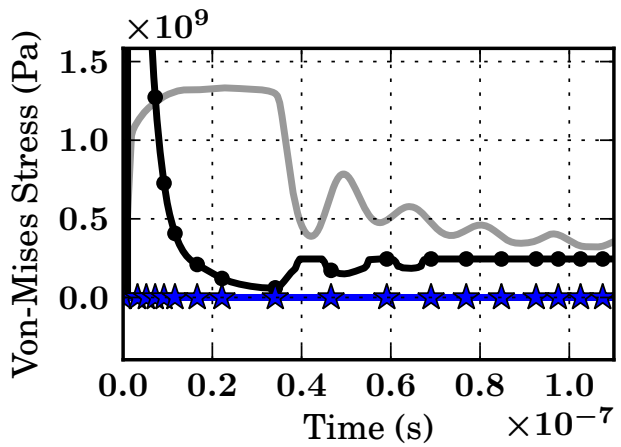

(c) Point $P_{0}$

Figure 15: Stress-Strain curves and stability analysis of various material points in a notched plate under impact 
The plots on the left side of figure 16 show the unstable elements predicted by the eigenvalue criterion at different times of the impact problem, and the plots on the right side show the corresponding equivalent plastic strain.

Figure $16 \mathrm{c}$ shows a snapshot of the instability region at the moment of the stress drop for $P_{0}$ and it is interesting to note that the unstable region is rapidly propagating horizontally. This seems to indicate that the unstable region defined by the eigenvalue criterion successfully predicts the propagation of the shear-band which in turn unloads $P_{0}$.

Figures $16 \mathrm{e}$ and $16 \mathrm{~g}$ show that the unstable region delineated by the eigenvalue criterion becomes narrower and shifts downward, which justifies why, according to the eigenvalue criterion, $P_{A}$ and $P_{B}$ are stable at $t=110 \mathrm{~ns}$. This happens because the deformation tends to concentrate and propagate from the bottom of the notch where there is a higher stress concentration.

\section{Concluding Remarks}

This work examined the stability of a thermal softening shear band model. It was shown that the instability point determined by applying the linear perturbation method to the model can be simplified to give the same criterion as Bai [26], extended to a non-linear Taylor Quinney coefficient. This result was then compared to a stability analysis by the indirect Lyapunov method applied to the consistent element Jacobian matrix of a finite element method, where matching results were found.

The instability point detected by both methods seems to determine the onset of the second stage of the deformation process as defined by Marchand and Duffy [73] where the strain distribution becomes inhomogeneous. As pointed out by Fressengeas and Molinari [29], instability by the linear perturbation method and intense localization do not have to occur simultaneously. This explains why linear instability and stress collapse are not correlated in the results showed in this work. 


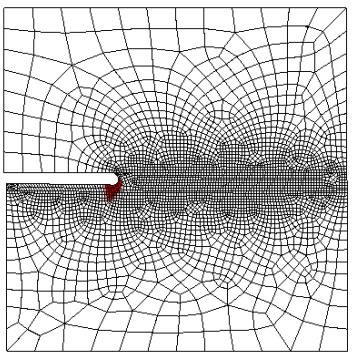

(a) Eigenvalue: $t=2 \mu \mathrm{s}$

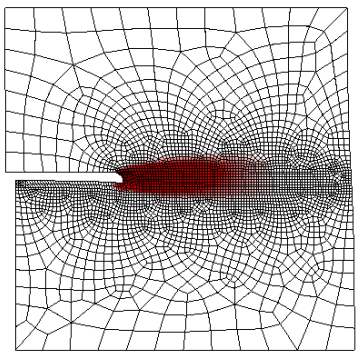

(c) Eigenvalue: $t=3.5 \mu \mathrm{s}$

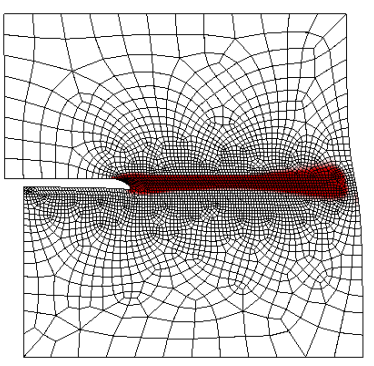

(e) Eigenvalue: $t=6.5 \mu \mathrm{s}$

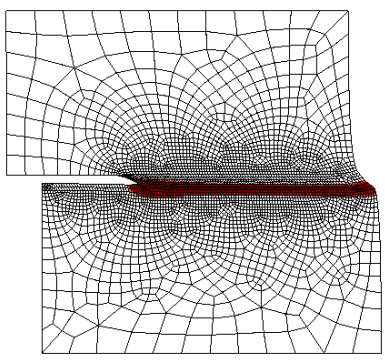

(g) Eigenvalue: $t=11 \mu \mathrm{s}$

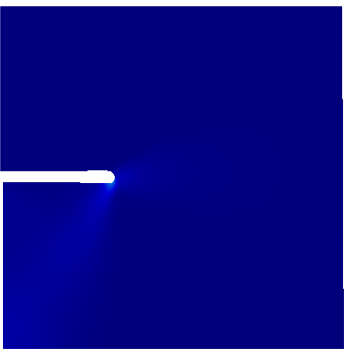

EQPS

5.0

4.0

2.0

0.0

(b) Eq. Plastic Strain: $t=2 \mu \mathrm{s}$

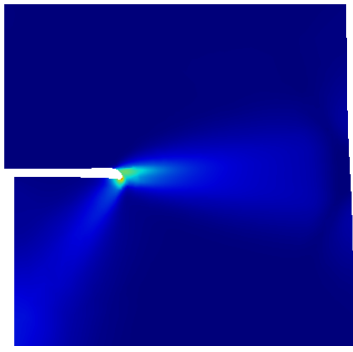

EQPS

5.0

4.0

2.0

0.0

(d) Eq. Plastic Strain: $t=3.5 \mu \mathrm{s}$

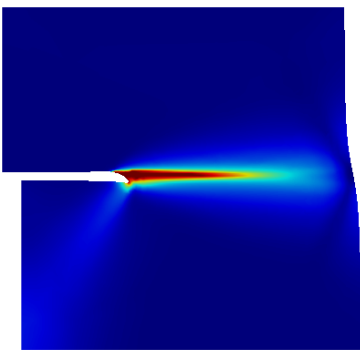

EQPS

5.0

4.0

2.0

0.0

(f) Eq. Plastic Strain: $t=6.5 \mu \mathrm{s}$

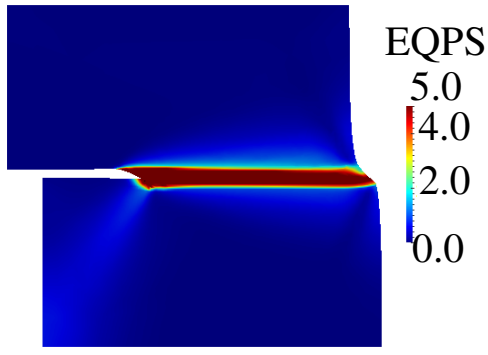

(h) Eq. Plastic Strain: $t=11 \mu \mathrm{s}$

Figure 16: Modeling of an impact onto a notched steel plate at different time snapshots. The plots on the left side show the prediction of element instability (colored in red) by the eigenvalue criterion and the plots on the right show the corresponding equivalent plastic strain (EQPS). 
The localized configuration corresponds to a bifurcated solution, which means that only in conditions that lead the solution to a bifurcation branch will the problem localize. An initial imperfection or asymmetry will tend to push the pre-localized solution in the direction of a particular bifurcation branch, which could possibly give a misleading idea that bifurcation/localization is already present. However, since the homogeneous (non-localized) solution has to become unstable before localization can occur and since the proposed methodology computes the point when the solution of the problem becomes unstable, it follows that this method predicts the point after which localization may occur.

The Indirect method of Lyapunov, or eigenvalue analysis of the consistent Jacobian matrix, presents as a good alternative methodology to study linear instability of shear bands. It possesses the advantage that it only requires the consistent Jacobian matrix that is used for the Newton iterations to solve the finite element problem, and therefore can be easily applied to more complex problems, as was exemplified for the case with non-linear Taylor-Quinney coefficient in 1D or the 2D examples. However, care should be taken to ensure that the instability modes detected are not associated with non-physical perturbation modes. Additionally, the eigenvalue analysis was shown to more accurately determine the unstable region undergoing localization when compared to the application of the analytical criterion derived for multidimensions but assuming adiabatic conditions.

It is also worth noting that the FEM implementation used in this work does not employ any special formulation that account for bifurcation. However, since this method successfully detects the propagation of instability, these results could be used to dynamically adapt the mesh or enhance the finite element formulation in these regions to obtain more accurate solutions or reduce the computational cost.

As predicted by the derivation presented, and exemplified with the non-linear Taylor-Quinney coefficient formulation used, the instability point will be affected by this non-linearity since the contribution of thermal softening to the flow-law is the main driver of the instability. 


\section{Acknowledgment}

This material is based upon work supported by the U.S. Department of Energy Office of Science, Office of Advanced Scientific Computing Research, Applied Mathematics program under Award Number DE-SC-0008196.

\section{Appendix A. Restriction of $\mathbf{J} 2$ formulation to $1 D$}

The shear strain is given by

$$
\gamma=\frac{\partial u}{\partial y}=2 \varepsilon_{12}
$$

The resultant stress tensor will only be non-zero for the deviatoric components in the $x-y$ direction. This deviatoric component will be defined as

$$
\tau=2 \mu \varepsilon_{12}=\mu \gamma
$$

which means that the second invariant

$$
J_{2}=\frac{1}{2} S: S=\tau^{2}
$$

and the effective stress

$$
\bar{\sigma}=\sqrt{3 J_{2}}=\sqrt{3}|\tau|
$$

Considering that the only non-zero term of the symmetric matrix $\dot{\varepsilon}^{p}$ is

$$
\dot{\varepsilon}_{12}^{p}=\frac{\gamma^{p}}{2}
$$

then

$$
\dot{\bar{\gamma}}_{p}=\sqrt{\frac{2}{3} \dot{\varepsilon}^{p}: \dot{\varepsilon}^{p}}=\frac{\dot{\gamma}^{p}}{\sqrt{3}}
$$


considering the non-linear characteristic equation for 1D as given by

$$
\tau=P(T) Q\left(\gamma^{p}\right) R\left(\dot{\gamma}^{p}\right) \Rightarrow \delta \tau=-P_{0} \delta T+Q_{0} \delta \gamma^{p}+R_{0} \delta \dot{\gamma}^{p}
$$

And the equation for $2 \mathrm{D}$ as

$$
\bar{\sigma}=p(T) q\left(\varepsilon^{p}\right) r\left(\dot{\varepsilon}^{p}\right) \Rightarrow \delta \bar{\sigma}=-p_{0} \delta T+q_{0} \delta \bar{\gamma}_{p}+r_{0} \delta \dot{\bar{\gamma}}_{p}
$$

Then

$$
\begin{gathered}
\delta \tau=\frac{\partial \tau}{\partial \bar{\sigma}} \delta \bar{\sigma} \\
P_{0}=p_{0} \frac{\partial \tau}{\partial \bar{\sigma}} \\
Q_{0}=q_{0} \frac{\partial \tau}{\partial \bar{\sigma}} \frac{\partial \bar{\gamma}_{p}}{\partial \gamma^{p}} \\
R_{0}=r_{0} \frac{\partial \tau}{\partial \bar{\sigma}} \frac{\partial \dot{\bar{\gamma}}_{p}}{\partial \dot{\gamma}^{p}}
\end{gathered}
$$

\section{Appendix B. Perturbation in 1D}

This appendix presents the full derivation of the characteristic equation obtained from perturbing the 1D set of governing equations and presented in section 2

Perturbing (17) gives:

$$
\rho c_{p} \delta \dot{T}=\lambda \frac{\partial^{2} \delta T}{\partial y^{2}}+\delta \chi \tau_{0} \dot{\gamma}_{0}^{p}+\chi_{0} \delta \tau \dot{\gamma}_{0}^{p}+\chi_{0} \tau_{0} \delta \dot{\gamma}^{p}
$$

Since $\chi$ is only a function of $\dot{\gamma}^{p}$, the previous equation can be rewritten as

$$
\rho c_{p} \delta \dot{T}=\lambda \frac{\partial^{2} \delta T}{\partial y^{2}}+\left(\left.\frac{\partial \chi}{\partial \dot{\gamma}^{p}}\right|_{0} \dot{\gamma}_{0}^{p}+\chi_{0}\right) \tau_{0} \delta \dot{\gamma}^{p}+\chi_{0} \dot{\gamma}_{0}^{p} \delta \tau
$$


Defining

$$
\chi_{0}=\chi^{*} \eta\left(\dot{\gamma}_{0}^{p}\right)=\chi^{*} \eta_{0},\left.\quad \frac{\partial \chi}{\partial \dot{\gamma}^{p}}\right|_{0}=\chi^{*} \eta^{\prime}\left(\dot{\gamma}_{0}^{p}\right)=\chi^{*} \eta_{0}^{\prime}
$$

and plugging back into $(\mathrm{B} .2)$ gives

$$
\rho c_{p} \delta \dot{T}=\lambda \frac{\partial^{2} \delta T}{\partial y^{2}}+\chi^{*}\left(\tau_{0}^{*} \delta \dot{\gamma}^{p}+\dot{\gamma}_{0}^{p *} \delta \tau\right)
$$

with

$$
\tau_{0}^{*}=\left(\eta_{0}^{\prime} \dot{\gamma}_{0}^{p}+\eta_{0}\right) \tau_{0}, \quad \dot{\gamma}_{0}^{p *}=\eta_{0} \dot{\gamma}_{0}^{p}
$$

Considering the formulation in $(20)$ for the perturbation $\delta T$ and taking the derivatives with respect to $t$ and $y$ gives:

$$
\left(\rho c_{p} \omega+k^{2} \lambda\right) \delta T=\chi^{*}\left(\tau_{0}^{*} \delta \dot{\gamma}^{p}+\delta \tau \dot{\gamma}_{0}^{p *}\right)
$$

Rearranging we obtain

$$
\delta T=K_{T}\left(\tau_{0}^{*} \delta \dot{\gamma}^{p}+\delta \tau \dot{\gamma}_{0}^{p *}\right)
$$

where

$$
K_{T}=\frac{\chi^{*}}{\rho c_{p} \omega+k^{2} \lambda}
$$

The perturbation of the non-linear constitutive equation (18) yields

$$
\delta \tau=-P_{0} \delta T+Q_{0} \delta \gamma^{p}+R_{0} \delta \dot{\gamma}^{p}
$$

where $P_{0}, Q_{0}$ and $R_{0}$ are defined by

$$
P_{0}=-\left.\frac{\partial \tau}{\partial T}\right|_{\mathbf{s}=\mathbf{s}_{0}} \quad ; \quad Q_{0}=\left.\frac{\partial \tau}{\partial \gamma^{p}}\right|_{\mathbf{s}=\mathbf{s}_{0}} \quad ; \quad R_{0}=\left.\frac{\partial \tau}{\partial \dot{\gamma}^{p}}\right|_{\mathbf{s}=\mathbf{s}_{0}}
$$

We note that $P_{0} \geq 0$ corresponds to thermal softening, $Q_{0} \geq 0$ corresponds to strain 
hardening and $R_{0} \geq 0$ corresponds to strain-rate hardening.

Substituting (B.7) into (B.9) gives:

$$
\delta \tau=-P_{0} K_{T}\left(\tau_{0}^{*} \delta \dot{\gamma}^{p}+\delta \tau \dot{\gamma}_{0}^{p *}\right)+Q_{0} \delta \gamma^{p}+R_{0} \delta \dot{\gamma}^{p}
$$

By perturbing (16) and its rate form, $\delta \gamma^{p}$ and $\delta \dot{\gamma}^{p}$ are plugged into (B.11), which yields

$$
\delta \tau=-P_{0} K_{T}\left[\tau_{0}^{*}\left(\delta \dot{\gamma}-\frac{\delta \dot{\tau}}{G}\right)+\delta \tau \dot{\gamma}_{0}^{p *}\right]+Q_{0}\left(\delta \gamma-\frac{\delta \tau}{G}\right)+R_{0}\left(\delta \dot{\gamma}-\frac{\delta \dot{\tau}}{G}\right)
$$

Rearranging Eq. (B.12), we get

$$
\delta \tau\left(1+\frac{Q_{0}}{G}+P_{0} \dot{\gamma}_{0}^{p *}\right)=\delta \gamma Q_{0}+\left(R_{0}-P_{0} K_{T} \tau_{0}^{*}\right)\left(\delta \dot{\gamma}-\frac{\delta \dot{\tau}}{G}\right)
$$

Differentiating both sides by $y$, using the momentum equation (15) and its rate form and plugging in the perturbation on $u$ gives

$$
\begin{aligned}
& \rho \omega^{2} \delta u\left(1+\frac{Q_{0}}{G}+P_{0} \dot{\gamma}_{0}^{p *}\right)= \\
& -k^{2} \delta u Q_{0}+\left(R_{0}-P_{0} K_{T} \tau_{0}^{*}\right)\left(-\omega k^{2} \delta u-\frac{\rho \omega^{3} \delta u}{G}\right)
\end{aligned}
$$

Equation $(\mathrm{B} .14)$ is expanded by plugging in $(\mathrm{B} .8)$ and eliminating $\delta u$, so that

$$
\begin{aligned}
& \left(\rho c_{p} \omega+k^{2} \lambda\right) \rho \omega^{2}\left(1+\frac{Q_{0}}{G}+P_{0} \dot{\gamma}_{0}^{p *}\right)=-\left(\rho c \omega+k^{2} \lambda\right) k^{2} Q_{0} \\
& +\left[\left(\rho c_{p} \omega+k^{2} \lambda\right) R_{0}-P_{0} \chi^{*} \tau_{0}^{*}\right]\left(-\omega k^{2}-\frac{\rho \omega^{3}}{G}\right)
\end{aligned}
$$

Finally, rearranging $\mathrm{B} .15$ in descending order for $\omega$, results in the following fourth-order characteristic equation $R(\omega)$

$$
R(\omega)=a_{4} \omega^{4}+a_{3} \omega^{3}+a_{2} \omega^{2}+a_{1} \omega+a_{0}=0
$$


where

$$
\begin{aligned}
& a_{0}=k^{4} \lambda Q_{0} \\
& a_{1}=k^{2}\left(k^{2} \lambda R_{0}+c_{p} Q_{0} \rho-\chi^{*} P_{0} \tau_{0}^{*}\right) \\
& a_{2}=\rho\left[\chi^{*} \dot{\gamma}_{0}^{p *} P_{0}+k^{2}\left(\lambda+c_{p} R_{0}\right)\right] \\
& a_{3}=\rho\left(c_{p} \rho+\frac{k^{2} \lambda R_{0}-\chi^{*} P_{0} \tau_{0}^{*}}{G}\right) \\
& a_{4}=\frac{c_{p} R_{0} \rho^{2}}{G}
\end{aligned}
$$

\section{Appendix C. Terms for FEM matrices of an element}

It is assumed that the reader is familiar with the concept of a shape function therefore it will not be defined here, for additional information on this topic any introductory level finite-element book should be sufficient. The variables $\mathbf{N}_{v}, \mathbf{N}_{\sigma}, \mathbf{N}_{T}$ and $\mathbf{N}_{\bar{\gamma}_{p}}$ represent the shape-functions for the velocity, stress, temperature and equivalent plastic strain fields, respectively.

The gradients of the shape functions are given by $\mathbf{B}_{v}, \mathbf{B}_{\sigma}, \mathbf{B}_{T}$ and $\mathbf{B}_{\bar{\gamma}_{p}}$ for velocity, stress, temperature and equivalent plastic strain fields, respectively. A noticeable exception is $\mathbf{B}_{v}^{s}$ which corresponds to the symmetric gradient of the velocity shape functions.

Appendix C.1. Mass Terms

$$
\begin{gathered}
\mathbf{M}_{v}=\int_{\Omega} \mathbf{N}_{v}^{T} \rho \mathbf{N}_{\dot{v}} \mathrm{~d} \Omega \\
\mathbf{M}_{\sigma}=\int_{\Omega} \mathbf{N}_{\sigma}^{T} \mathbf{N}_{\dot{\sigma}} \mathrm{d} \Omega \\
\mathbf{M}_{T}=\int_{\Omega} \mathbf{N}_{T}^{T} \rho c \mathbf{N}_{\dot{T}} \mathrm{~d} \Omega \\
\mathbf{M}_{\bar{\gamma}_{p}}=\int_{\Omega} \mathbf{N}_{\bar{\gamma}_{p}}^{T} \mathbf{N}_{\dot{\gamma}_{p}} \mathrm{~d} \Omega
\end{gathered}
$$


Note that the shape functions for the rate of the fields are the same as the shape functions used for the fields themselves. This is due to the fact that, after the discretization in time, the Jacobian matrix will only depend upon the field quantities, and not their rate.

Appendix C.2. Linear Terms

$$
\begin{gathered}
\mathbf{K}_{v \sigma}=-\int_{\Omega} \mathbf{B}_{v}^{T} \mathbf{N}_{\sigma} \mathrm{d} \Omega \\
\mathbf{K}_{\sigma v}=\int_{\Omega} \mathbf{N}_{\sigma}^{T} \mathbf{C}^{\text {elas }}: \mathbf{B}_{v}^{s} \mathrm{~d} \Omega \\
\mathbf{K}_{T T}=-\int_{\Omega} \mathbf{B}_{T}^{T} \lambda \mathbf{B}_{T} \mathrm{~d} \Omega
\end{gathered}
$$

Appendix C.3. Non-linear Terms

$$
\begin{gathered}
\mathbf{G}_{\sigma \sigma}=-\int_{\Omega} \mathbf{N}_{\sigma}^{T} \mathbf{C}^{\text {elas }}:\left[\frac{3}{2 \bar{\sigma}} \frac{\partial g}{\partial \bar{\sigma}} \frac{\partial \bar{\sigma}}{\partial \sigma} \mathbf{S}+\frac{3 g}{2} \frac{\partial}{\partial \sigma}\left(\frac{\mathbf{S}}{\bar{\sigma}}\right)\right] \mathbf{N}_{\sigma} \mathrm{d} \Omega \\
\mathbf{G}_{\sigma T}=-\int_{\Omega} \mathbf{N}_{\sigma}^{T} \mathbf{C}^{\text {elas }}:\left[\frac{3}{2 \bar{\sigma}} \frac{\partial g}{\partial T} \mathbf{S}\right] \mathbf{N}_{T} \mathrm{~d} \Omega \\
\mathbf{G}_{\sigma \bar{\gamma}_{p}}=-\int_{\Omega} \mathbf{N}_{\sigma}^{T} \mathbf{C}^{\text {elas }}:\left[\frac{3}{2 \bar{\sigma}} \frac{\partial g}{\partial \bar{\gamma}_{p}} \mathbf{S}\right] \mathbf{N}_{\bar{\gamma}_{p}} \mathrm{~d} \Omega \\
\mathbf{G}_{\bar{\gamma}_{p} \sigma}=\int_{\Omega} \mathbf{N}_{\bar{\gamma}_{p}}^{T} \frac{\partial g}{\partial \bar{\sigma}} \frac{\partial \bar{\sigma}}{\partial \sigma} \mathbf{N}_{\sigma} \mathrm{d} \Omega \\
\mathbf{G}_{\bar{\gamma}_{p} T}=\int_{\Omega} \mathbf{N}_{\bar{\gamma}_{p}}^{T} \frac{\partial g}{\partial T} \mathbf{N}_{T} \mathrm{~d} \Omega
\end{gathered}
$$




$$
\mathbf{G}_{\bar{\gamma}_{p} \bar{\gamma}_{p}}=\int_{\Omega} \mathbf{N}_{\bar{\gamma}_{p}}^{T} \frac{\partial g}{\partial \bar{\gamma}_{p}} \mathbf{N}_{\bar{\gamma}_{p}} \mathrm{~d} \Omega
$$




\section{References}

[1] D. Bigoni. Bifurcation and instability of non-associative elastoplastic solids. In Material Instabilities in Elastic and Plastic Solids, Petryk H, pages 1-52. Springer, 2000.

[2] A. Gajo, D. Bigoni, and D.Muir Wood. Multiple shear band development and related instabilities in granular materials. Journal of the Mechanics and Physics of Solids, 52(12): 2683-2724, 2004. ISSN 0022-5096. doi: 10.1016/j.jmps.2004.05.010.

[3] Shaofan Li, Wing Kam Liu, Ares J. Rosakis, Ted Belytschko, and Wei Hao. Mesh-free galerkin simulations of dynamic shear band propagation and failure mode transition. International Journal of Solids and Structures, 39(5):1213-1240, 2002. ISSN 0020-7683. doi: 10.1016/S0020-7683(01)00188-3.

[4] M. Zhou, G. Ravichandran, and A.J. Rosakis. Dynamically propagating shear bands in impact-loaded prenotched plates-II. numerical simulations. Journal of the Mechanics and Physics of Solids, 44(6):1007-1032, 1996. ISSN 0022-5096. doi: 10.1016/0022-5096(96) 00004-X.

[5] M. Zhou, A. Needleman, and R.J. Clifton. Finite element simulations of shear localization in plate impact. Journal of the Mechanics and Physics of Solids, 42(3):423-458, 1994. ISSN 0022-5096. doi: 10.1016/0022-5096(94)90026-4.

[6] Ted Belytschko, Huai-Yang Chiang, and Edward Plaskacz. High resolution two-dimensional shear band computations: imperfections and mesh dependence. Computer Methods in Applied Mechanics and Engineering, 119(1-2):1-15, 1994. ISSN 0045-7825. doi: 10.1016/ 0045-7825(94)00073-5.

[7] R.C. Batra. The initiation and growth of, and the interaction among, adiabatic shear bands in simple and dipolar materials. International Journal of Plasticity, 3(1):75-89, 1987. ISSN 0749-6419. doi: 10.1016/0749-6419(87)90019-2.

[8] R.C. Batra and L. Chen. Effect of viscoplastic relations on the instability strain, shear band initiation strain, the strain corresponding to the minimum shear band spacing, and the band width in a thermoviscoplastic material. International Journal of Plasticity, 17(11):14651489, 2001. ISSN 0749-6419. doi: 10.1016/S0749-6419(01)00004-3.

[9] T.W. Wright and R.C. Batra. The initiation and growth of adiabatic shear bands. International Journal of Plasticity, 1(3):205-212, 1985. ISSN 0749-6419. doi: 10.1016/ 0749-6419(85)90003-8. 
[10] E. C. Aifantis. On the microstructural origin of certain inelastic models. Journal of Engineering Materials and Technology, 106(4):326-330, 1984. ISSN 0094-4289. doi: 10.1115/1.3225725.

[11] Elias C. Aifantis. The physics of plastic deformation. International Journal of Plasticity, 3 (3):211-247, 1987. ISSN 0749-6419. doi: 10.1016/0749-6419(87)90021-0.

[12] Rashid K. Abu Al-Rub and George Z. Voyiadjis. A physically based gradient plasticity theory. International Journal of Plasticity, 22(4):654-684, 2006. ISSN 0749-6419. doi: 10.1016/j.ijplas.2005.04.010.

[13] Rashid K. Abu Al-Rub and George Z. Voyiadjis. A finite strain plastic-damage model for high velocity impact using combined viscosity and gradient localization limiters: Part i theoretical formulation. International Journal of Damage Mechanics, 15(4):293-334, 2006. ISSN 1056-7895, 1530-7921. doi: 10.1177/1056789506058046.

[14] George Z. Voyiadjis and Rashid K. Abu Al-Rub. A finite strain plastic-damage model for high velocity impacts using combined viscosity and gradient localization limiters: Part II - numerical aspects and simulations. International Journal of Damage Mechanics, 15(4): 335-373, 2006. ISSN 1056-7895, 1530-7921. doi: 10.1177/1056789506058047.

[15] E. N. Lages, G. H. Paulino, I. F. M. Menezes, and R. R. Silva. Nonlinear finite element analysis using an object-oriented philosophy - application to beam elements and to the cosserat continuum. Engineering with Computers, 15(1):73-89, 1999. ISSN 0177-0667, 1435-5663. doi: 10.1007/s003660050006.

[16] A. Cemal Eringen. Nonlocal Continuum Field Theories. Springer New York, 2002. ISBN 9780387952758 .

[17] Zdenek P Bažant and Milan Jirásek. Nonlocal integral formulations of plasticity and damage: survey of progress. Journal of Engineering Mechanics, 128(11):1119-1149, 2002.

[18] R.H.J. Peerlings, M.G.D. Geers, R. de Borst, and W.A.M. Brekelmans. A critical comparison of nonlocal and gradient-enhanced softening continua. International Journal of Solids and Structures, 38(44-45):7723-7746, 2001. ISSN 0020-7683. doi: 10.1016/S0020-7683(01) 00087-7.

[19] Shaofan Li, Wei Hao, and Wing Kam Liu. Mesh-free simulations of shear banding in large deformation. International Journal of Solids and Structures, 37(48-50):7185-7206, 2000. ISSN 0020-7683. doi: 10.1016/S0020-7683(00)00195-5. 
[20] Shaofan Li and Wing Kam Liu. Numerical simulations of strain localization in inelastic solids using mesh-free methods. International Journal for Numerical Methods in Engineering, 48(9):1285-1309, 2000.

[21] Luc Berger-Vergiat, Colin McAuliffe, and Haim Waisman. Isogeometric analysis of shear bands. Computational Mechanics, pages 1-19, 2014. ISSN 0178-7675, 1432-0924. doi: 10.1007/s00466-014-1002-8.

[22] Colin McAuliffe and Haim Waisman. Mesh insensitive formulation for initiation and growth of shear bands using mixed finite elements. Computational Mechanics, 51(5):807-823, 2013. ISSN 0178-7675, 1432-0924. doi: 10.1007/s00466-012-0765-z.

[23] E. A. Burroughs, L. A. Romero, R. B. Lehoucq, and A. G. Salinger. Large scale eigenvalue calculations for computing the stability of buoyancy driven flows. Technical report, 2001.

[24] H. Mittelmann, K. Chang, and D. Jankowski. Iterative solution of the eigenvalue problem in hopf bifurcation for the boussinesq equations. SIAM Journal on Scientific Computing, 15 (3):704-712, 1994. ISSN 1064-8275. doi: 10.1137/0915045.

[25] L. Anand, K.H. Kim, and T.G. Shawki. Onset of shear localization in viscoplastic solids. Journal of the Mechanics and Physics of Solids, 35(4):407-429, 1987. ISSN 0022-5096. doi: 10.1016/0022-5096(87)90045-7.

[26] Y.L. Bai. Thermo-plastic instability in simple shear. Journal of the Mechanics and Physics of Solids, 30(4):195-207, 1982. ISSN 0022-5096. doi: 10.1016/0022-5096(82)90029-1.

[27] R.C. Batra and Z.G. Wei. Instability strain and shear band spacing in simple tensile/compressive deformations of thermoviscoplastic materials. International Journal of Impact Engineering, 34(3):448-463, 2007. ISSN 0734-743X. doi: 10.1016/j.ijimpeng.2005. 11.004 .

[28] L.H. Dai and Y.L. Bai. Basic mechanical behaviors and mechanics of shear banding in BMGs. International Journal of Impact Engineering, 35(8):704-716, 2008. ISSN 0734743X. doi: 10.1016/j.ijimpeng.2007.10.007.

[29] C. Fressengeas and A. Molinari. Instability and localization of plastic flow in shear at high strain rates. Journal of the Mechanics and Physics of Solids, 35(2):185-211, 1987. ISSN 0022-5096. doi: 10.1016/0022-5096(87)90035-4. 
[30] Xianwu Ling and T. Belytschko. Thermal softening induced plastic instability in ratedependent materials. Journal of the Mechanics and Physics of Solids, 57(4):788-802, 2009. ISSN 0022-5096. doi: 10.1016/j.jmps.2008.04.010.

[31] Jwo Pan. Perturbation analysis of shear strain localization in rate sensitive materials. International Journal of Solids and Structures, 19(2):153-164, 1983. ISSN 0020-7683. doi: 10.1016/0020-7683(83)90006-9.

[32] T.W. Wright. Shear band susceptibility: Work hardening materials. International Journal of Plasticity, 8(5):583-602, 1992. ISSN 0749-6419. doi: 10.1016/0749-6419(92)90032-8.

[33] J. N. Baucom and M. A. Zikry. Perturbation analysis of high strain-rate shear localization in B.C.C. crystalline materials. Acta Mechanica, 137(1-2):109-129, 1999. ISSN 0001-5970, 1619-6937. doi: 10.1007/BF01313148.

[34] Kwon Hee Kim. Shear Localization in Viscoplastic Solids. Ph.D. dissertation, Department of Mechanical Engineering, Massachusetts Institute of Technology, 1987.

[35] D Bigoni and D Zaccaria. On the eigenvalues of the acoustic tensor in elastoplasticity. Eur. J. Mech., A/Solids, 13(5):621-638, 1994.

[36] Ronaldo I. Borja. Bifurcation of elastoplastic solids to shear band mode at finite strain. Computer Methods in Applied Mechanics and Engineering, 191(46):5287-5314, 2002. ISSN 0045-7825. doi: 10.1016/S0045-7825(02)00459-0.

[37] L. Szabó. On the eigenvalues of the fourth-order constitutive tensor and loss of strong ellipticity in elastoplasticity. International Journal of Plasticity, 13(10):809-835, 1997. ISSN 0749-6419. doi: 10.1016/S0749-6419(97)00067-3.

[38] Liang Xue and Ted Belytschko. Fast methods for determining instabilities of elastic-plastic damage models through closed-form expressions. International Journal for Numerical Methods in Engineering, 84(12):1490-1518, 2010. ISSN 1097-0207. doi: 10.1002/nme.2947.

[39] J. Lemonds and A. Needleman. Finite element analyses of shear localization in rate and temperature dependent solids. Mechanics of Materials, 5(4):339-361, 1986. ISSN 01676636. doi: 10.1016/0167-6636(86)90039-6.

[40] J. J. Mason, A. J. Rosakis, and G. Ravichandran. On the strain and strain rate dependence of the fraction of plastic work converted to heat: an experimental study using high speed infrared detectors and the kolsky bar. Mechanics of Materials, 17(2-3):135-145, 1994. ISSN 0167-6636. doi: 10.1016/0167-6636(94)90054-X. 
[41] P. Rosakis, A. J. Rosakis, G. Ravichandran, and J. Hodowany. A thermodynamic internal variable model for the partition of plastic work into heat and stored energy in metals. Journal of the Mechanics and Physics of Solids, 48(3):581 - 607, 2000. ISSN 0022-5096. doi: http://dx.doi.org/10.1016/S0022-5096(99)00048-4.

[42] N. Aravas, K-S. Kim, and F. A. Leckie. On the calculations of the stored energy of cold work. Journal of Engineering Materials and Technology, 112(4):465-470, 1990. ISSN 0094-4289. doi: $10.1115 / 1.2903358$.

[43] M. Vural, G. Ravichandran, and D. Rittel. Large strain mechanical behavior of 1018 coldrolled steel over a wide range of strain rates. Metallurgical and Materials Transactions A, 34(12):2873-2885, 2003. ISSN 1073-5623, 1543-1940. doi: 10.1007/s11661-003-0188-8.

[44] J. C. Simo and T. J. R. Hughes. Computational Inelasticity. Springer, 1998. ISBN 9780387975207.

[45] G. I. Taylor and H. Quinney. The latent energy remaining in a metal after cold working. Proceedings of the Royal Society of London. Series A, 143(849):307-326, 1934. ISSN 13645021, 1471-2946. doi: 10.1098/rspa.1934.0004.

[46] Anis Hor, Franck Morel, Jean-Lou Lebrun, and Guénaël Germain. Modelling, identification and application of phenomenological constitutive laws over a large strain rate and temperature range. Mechanics of Materials, 64:91 - 110, 2013. ISSN 0167-6636. doi: http://dx.doi.org/10.1016/j.mechmat.2013.05.002.

[47] Gordon R. Johnson and William H. Cook. Fracture characteristics of three metals subjected to various strains, strain rates, temperatures and pressures. Engineering Fracture Mechanics, 21(1):31 - 48, 1985. ISSN 0013-7944. doi: http://dx.doi.org/10.1016/0013-7944(85) 90052-9.

[48] A.G. Odeshi, M.N. Bassim, S. Al-Ameeri, and Q. Li. Dynamic shear band propagation and failure in AISI 4340 steel. Journal of Materials Processing Technology, 169(2):150-155, 2005. ISSN 0924-0136. doi: 10.1016/j.jmatprotec.2005.03.016.

[49] V.E Howle and Lloyd N Trefethen. Eigenvalues and musical instruments. Journal of Computational and Applied Mathematics, 135(1):23-40, 2001. ISSN 0377-0427. doi: 10.1016/S0377-0427(00)00560-4.

[50] Richard M. Murray, Zexiang Li, and S. Shankar Sastry. A Mathematical Introduction to Robotic Manipulation. CRC Press, 1994. ISBN 9780849379819. 
[51] Edward John Routh. A Treatise on the Stability of a Given State of Motion: Particularly Steady Motion. Macmillan and Company, 1877.

[52] A. Hurwitz. Ueber die bedingungen, unter welchen eine gleichung nur wurzeln mit negativen reellen theilen besitzt. Mathematische Annalen, 46:273-284, 1895. ISSN 0025-5831; 14321807.

[53] A. M. Lyapunov. General Problem of the Stability Of Motion. CRC Press, 1 edition, 1992.

[54] Guanrong Chen. Stability of nonlinear systems. In Wiley Encyclopedia of Electrical and Electronics Engineering, pages 4881-4896. John Wiley \& Sons, Inc., 2001. ISBN 9780471346081 .

[55] Prof Ivo Babuška. Error-bounds for finite element method. Numerische Mathematik, 16(4): 322-333, 1971. ISSN 0029-599X, 0945-3245. doi: 10.1007/BF02165003.

[56] F. Brezzi. On the existence, uniqueness and approximation of saddle-point problems arising from lagrangian multipliers. ESAIM: Mathematical Modelling and Numerical Analysis Modélisation Mathématique et Analyse Numérique, 8(R2):129-151, 1974.

[57] E. Reissner. On a variational theorem in elasticity. Journal of Mathematical Physics, 29(2): 90-95, 1950.

[58] Jorg F. Kalthoff. Shadow optical analysis of dynamic shear fracture. Optical Engineering, 27(10):835-840, 1988. ISSN 0091-3286. doi: 10.1117/12.7976772.

[59] R. L. Taylor. FEAP - finite element analysis program, 2014.

[60] Satish Balay, Jed Brown, Kris Buschelman, Victor Eijkhout, William D. Gropp, Dinesh Kaushik, Matthew G. Knepley, Lois Curfman McInnes, Barry F. Smith, and Hong Zhang. PETSc users manual. Technical Report ANL-95/11 - Revision 3.3, Argonne National Laboratory, 2012.

[61] E. Anderson, Z. Bai, C. Bischof, S. Blackford, J. Demmel, J. Dongarra, J. Du Croz, A. Greenbaum, S. Hammarling, A. McKenney, and D. Sorensen. LAPACK Users' Guide. Society for Industrial and Applied Mathematics, Philadelphia, PA, third edition, 1999. ISBN 0-89871-447-8 (paperback).

[62] T.W. Wright. Approximate analysis for the formation of adiabatic shear bands. Journal of the Mechanics and Physics of Solids, 38(4):515-530, 1990. ISSN 0022-5096. doi: 10.1016/ 0022-5096(90)90012-S. 
[63] Bertram Hopkinson. A method of measuring the pressure produced in the detonation of high explosives or by the impact of bullets. Philosophical Transactions of the Royal Society of London. Series A, Containing Papers of a Mathematical or Physical Character, 213(497508):437-456, 1914. ISSN 1364-503X, 1471-2962. doi: 10.1098/rsta.1914.0010.

[64] H. Kolsky. An investigation of the mechanical properties of materials at very high rates of loading. Proceedings of the Physical Society. Section B, 62(11):676-700, 1949. ISSN 0370-1301. doi: 10.1088/0370-1301/62/11/302.

[65] Weinong W. Chen and Bo Song. Split Hopkinson (Kolsky) Bar: Design, Testing and Applications. Springer, 2010. ISBN 9781441979827.

[66] T. W. Wright and J. W. Walter. On stress collapse in adiabatic shear bands. Journal of the Mechanics and Physics of Solids, 35(6):701-720, 1987. ISSN 0022-5096. doi: 10.1016/ 0022-5096(87)90051-2.

[67] R.C. Batra and C.H. Kim. Analysis of shear banding in twelve materials. International Journal of Plasticity, 8(4):425-452, 1992. ISSN 0749-6419. doi: 10.1016/0749-6419(92) 90058-K.

[68] B. Dodd and Y. Bai. Width of adiabatic shear bands. Materials Science and Technology, 1 (1):38-40, 1985. ISSN 0267-0836. doi: 10.1179/mst.1985.1.1.38.

[69] Colin McAuliffe and Haim Waisman. A Pian-Sumihara type element for modeling shear bands at finite deformation. Computational Mechanics, 53(5):925-940, 2014. ISSN 01787675, 1432-0924. doi: 10.1007/s00466-013-0940-x.

[70] Zdeněk P Bažant. Structural stability. International Journal of Solids and Structures, 37 (1-2):55-67, 2000. ISSN 0020-7683. doi: 10.1016/S0020-7683(99)00078-5.

[71] R.C. Batra, N.A. Jaber, and M.E. Malsbury. Analysis of failure modes in an impact loaded thermoviscoplastic prenotched plate. International Journal of Plasticity, 19(2):139-196, 2003. ISSN 0749-6419. doi: 10.1016/S0749-6419(01)00032-8.

[72] R. C. Batra and M. V. S. Ravinsankar. Three-dimensional numerical simulation of the kalthoff experiment. International Journal of Fracture, 105(2):161-186, 2000. ISSN 03769429, 1573-2673. doi: 10.1023/A:1007658224458.

[73] A. Marchand and J. Duffy. An experimental study of the formation process of adiabatic shear bands in a structural steel. Journal of the Mechanics and Physics of Solids, 36(3):251-283, 1988. ISSN 0022-5096. doi: 10.1016/0022-5096(88)90012-9. 